\title{
Probes of the quark-gluon plasma and plasma instabilities
}

\author{
Sigtryggur Hauksson, ${ }^{*}$ Sangyong Jeon, and Charles Gale \\ Department of Physics, McGill University, 3600 University Street, Montréal, QC, Canada H3A 2T8
}

(Received 16 December 2020; revised 11 March 2021; accepted 25 May 2021; published 8 June 2021)

\begin{abstract}
Penetrating probes in heavy-ion collisions, like jets and photons, are sensitive to the transport coefficients of the produced quark-gluon plasma, such as shear and bulk viscosity. Quantifying this sensitivity requires a detailed understanding of photon emission and jet-medium interaction in a nonequilibrium plasma during the hydrodynamic stages of heavy-ion collisions. Up to now, such an understanding has been hindered by plasma instabilities. These instabilities arise out of equilibrium and lead to spurious divergences when evaluating the rate of interaction of hard probes with the plasma. In this paper, we show that taking into account the time evolution of an unstable plasma cures these divergences. Specifically, we calculate the time evolution of gluon two-point correlators in a setup with a small initial momentum anisotropy and show that the gluon occupation density at first grows exponentially. Based on this calculation, we argue for a phenomenological prescription where instability poles are subtracted. Finally, we show that in the Abelian case instability fields do not affect medium-induced photon emission to our order of approximation.
\end{abstract}

DOI: 10.1103/PhysRevC.103.064904

\section{INTRODUCTION}

In heavy-ion collisions at ultrarelativistic energies a dense medium of quarks and gluons is formed: the quark-gluon plasma (QGP) [1]. The medium expands and cools until the quarks and gluons coalesce into soft hadrons which rescatter and fly to the detectors. Remarkably, this time evolution of the QGP is captured by hydrodynamics making the QGP a relativistic fluid [2]. A major goal of heavy-ion collision experiments is to characterize this QGP using transport coefficients, such as shear and bulk viscosity, which quantify its response to weak perturbations and are fundamental properties of QCD. Hydrodynamic studies have shown that the ratio of shear viscosity to entropy density of QGP is the lowest of any known material [2], but arguably the precise value is only known within a factor of 2 or so. Another major goal of these experiments is to understand how the QGP is formed in the first place. Specifically, it needs to be understood how an initial collision of two heavy nuclei at high energies gives rise to a macroscopic fluid within a time frame of $1 \mathrm{fm} / \mathrm{c}$ or even less.

Explaining equilibration and transport coefficients in heavy-ion collisions relies on knowledge of the nonequilibrium physics of the quark-gluon plasma. Up until now transport coefficients of the QGP have mostly been extracted

*Corresponding author: sigtryggur.hauksson@mail.mcgill.ca

Published by the American Physical Society under the terms of the Creative Commons Attribution 4.0 International license. Further distribution of this work must maintain attribution to the author(s) and the published article's title, journal citation, and DOI. Funded by $S C O A P^{3}$. by fitting hydrodynamic studies to experimental results of the yield and angular distribution of soft hadrons [2]. An alternative way is offered by hard probes of the QGP, such as photons, jets, and heavy quarks. As an example, jets broaden and lose energy as they interact with the QGP medium [3]. The rate of interaction and its dependence on the energy of a jet particle depend in detail on the makeup of the fluid. Thus the interaction with a thermally equilibrated fluid and a fluid with shear flow will be different, meaning that the energy loss of jets is sensitive to the QGP's shear viscosity [4]. To use jets or photons to get the QGP's shear viscosity requires thorough understanding of hard probes in nonequilibrium QGP.

In this paper we focus on hard probes in the nonequilibrium plasma present in the hydrodynamic stage of heavy-ion collisions. A number of challenges arise when calculating nonequilibrium effects on hard probes in the plasma. An important challenge comes from instabilities intrinsic to weakly coupled plasmas. These Weibel instabilities $[5,6]$ come about when quasiparticles that are anisotropically distributed in momentum space radiate soft gluons, the density of which grows exponentially with time. The system is thus intrinsically time dependent. The effect of this time dependence on mediuminduced processes in hard probes has not been taken into account. ${ }^{1}$ Taking it into account is essential since otherwise spurious divergences arise, such as in the rate of jet particles splitting or the rate of photon production in nonequilibrium QGP [9-12].

\footnotetext{
${ }^{1}$ We note that jet broadening and energy loss due to acceleration of partons in the unstable fields have been studied and do not suffer from these divergences $[7,8]$.
} 
This paper is organized as follows: In Sec. II we explain how instabilities in weakly coupled QGP lead to spurious divergences when studying hard probes in a nonequilibrium plasma. In Secs. III and IV we calculate the time evolution of gluon correlators in a nonequilibrium plasma with slight initial momentum anisotropy. We argue that for phenomenological applications the contribution of instabilities should be subtracted. Finally, in Secs. V and VI we show that in the Abelian case instability fields do not affect medium-induced photon emission to our order of approximation. Details of calculations are relocated to the Appendices.

\section{BACKGROUND}

Instabilities in a weakly coupled nonequilibrium quarkgluon plasma lead to spurious divergences when calculating, e.g., the rate of photon emission from the plasma or the rate of jet-medium interaction. Understanding the origin of these divergences requires some background on weakly coupled plasmas and quantum-field theoretical calculations of photon emission.

The ultimate goal when calculating photon emission in a nonequilibrium plasma is to learn about the QGP formed in experiments by using photons. This necessitates a flexible approach where rates of photon production can be combined with hydrodynamic simulations of heavy-ion collisions. Specifically, we have two conditions.

(1) The rate of photon production should only depend on the properties of the medium in that instant, and not on the medium's history. This requires

$$
t_{\text {process }} \ll t_{\text {medium }}
$$

where $t_{\text {process }}$ is the time it takes to emit a photon and $t_{\text {medium }}$ is the time scale over which the medium changes substantially.

(2) The rate of photon production should depend solely on macroscopic variables, like pressure and shear flow, that can be obtained from hydrodynamic calculations. This is achieved by describing the medium by a quasiparticle momentum distribution $f(\mathbf{p})$ that corresponds to the macroscopic variables. ${ }^{2}$

These two conditions have immediate consequences for quantum field theory calculations of photon production. The first condition says that the medium is effectively static during the emission of a photon. We thus want to specify a quasiparticle distribution $f(\mathbf{p})$ at an initial time $t_{0} \rightarrow-\infty$ which will appear in bare propagators. Assuming that $f(\mathbf{p})$ remains the same during photon emission, we can use the same bare propagators at all times. Since time ranges from $-\infty$ to $\infty$ we can do Fourier transforms and work in frequency space which provides huge simplification. Naively, we expect the results for the rate of photon production to have the same form as in

\footnotetext{
${ }^{2}$ In general, there might be multiple momentum distributions for the same macroscopic variables but the hope is that the calculation is not sensitive to which distribution is chosen as long as the macroscopic variables remain the same.
}

thermal equilibrium, with equilibrium distributions replaced by a more general distribution $f(\mathbf{p}) .^{3}$

Unfortunately, this simple picture does not work in general. As explained in greater detail below, one generally gets a nonsensical, infinite rate of photon production when assuming a static medium characterized by a momentum distribution $f(\mathbf{p})$. The culprits are instabilities in the plasma which give rise to rapid exponential growth in the density of soft gluons, violating the assumption of a static medium. These instabilities arise for any momentum distribution that is anisotropic, i.e., $f(\mathbf{p}) \neq f(p)$. (In the case of thermal equilibrium or other isotropic distributions the instabilities are not present and one can assume a static medium.) In fact, the same problem of divergent rates is present when calculating, e.g., the rate of jet-medium interaction $[9,10]$, heavy-quark potential [11], and even the rate of interaction among the quasiparticles comprising the medium [12].

Understanding this problem better requires a detailed discussion of weakly coupled QCD plasmas that are sufficiently close to equilibrium. Such plasmas are characterized by two energy scales. First, there are quasiparticles-quarks and gluons - which are localized and propagate freely, apart from occasionally interacting with each other. Their phase space behavior can be described by kinetic theory [12], and their distribution functions obey a Boltzmann equation:

$$
v^{\mu} \frac{\partial f}{\partial x^{\mu}}+\mathbf{F} \cdot \frac{\partial f}{\partial \mathbf{p}}=\bar{C}[f, A]
$$

where the distribution $f(t, \mathbf{x} ; \mathbf{p})$ changes because of external forces $\mathbf{F}$ and collisions between quasiparticles, as described by $\bar{C}$. Here color indices have been suppressed for simplicity.

The quasiparticles radiate gluon fields with energy $g \Lambda$ where $g \ll 1$ is the coupling constant. These long-wavelength, soft gluons have high occupancy and can thus be described using classical field theory. Specifically, they obey the classical equations of motion for a gluon field $A^{\mu}$ :

$$
\mathcal{D}_{\mu} F^{\mu \nu}=j^{\nu},
$$

where $\mathcal{D}_{\mu}$ is a covariant derivative, $F^{\mu \nu}$ is the chromoelectromagnetic tensor, and $j^{\mu}$ is a current which comes from the quark and gluon quasiparticles.

These two coupled equations, Eqs. (2) and (3), tell us that quasiparticles source gluon fields which deflect the quasiparticles in turn. They can be solved simultaneously, giving rise to an effective field theory for the long-wavelength gluons called hard thermal loops (HTL) [15]. We write the quasiparticle momentum distribution as

$$
f(\mathbf{p})=f_{0}(\mathbf{p})+\delta f\left(x^{\mu} ; \mathbf{p}\right)
$$

where $\delta f$ is a small fluctuation around the distribution $f_{0}$ specified at the initial time $t_{0} \rightarrow-\infty$. Dropping the subleading

\footnotetext{
${ }^{3} \mathrm{~A}$ detailed argument is needed to show this [13], as the original calculation of leading-order photon production in a plasma assumed the Kubo-Martin-Schwinger condition which is only valid in thermal equilibrium [14].
} 
collision kernel, Eq. (2) then becomes

$$
v^{\mu} \frac{\partial \delta f}{\partial x^{\mu}}=-\mathbf{F} \cdot \frac{\partial f_{0}}{\partial \mathbf{p}}
$$

where an external force $\mathbf{F}\left[A^{\mu}\right]$ due to an applied gauge field $A^{\mu}$ sources fluctuation $\delta f$. Solving for the fluctuation gives a current $j^{\mu}\left[A^{\mu}\right] \sim \int d^{3} p \frac{p^{\mu}}{p} \delta f$ which linear response theory tells us is related to the applied field $A^{\mu}$ through $j(P)=$ $\Pi_{\text {ret }}(P) A(P)$. We thus get the retarded self-energy for soft gluons [16]:

$$
\begin{aligned}
\Pi_{\mathrm{ret}}^{\mu \nu}(Q) \sim & g^{2} \int \frac{d^{3} p}{2 p(2 \pi)^{3}} f_{0}(\mathbf{p}) \\
\times & {\left.\left[g^{\mu \nu}-Q \cdot \partial_{P} \frac{P^{\mu} P^{\nu}}{P \cdot Q-i \epsilon}\right]\right|_{p^{0}=p}, }
\end{aligned}
$$

which depends explicitly on the initial momentum distribution $f_{0}$. Here $P$ and $Q$ are four-momenta while $p=|\mathbf{p}|$ is the threemomentum.

Equation (6) contains a wealth of information on how soft gluons propagate in the medium. Continuing with the assumption of a static medium, the retarded propagator $G_{\mathrm{ret}}^{\mu \nu}(x, y)=$ $\theta\left(t_{x}-t_{y}\right)\left\langle\left[A^{\mu}, A^{\nu}\right]\right\rangle$ becomes

$$
G_{\text {ret }}(P)=i\left[P^{2}-\Pi_{\mathrm{ret}}(P)\right]^{-1}
$$

in momentum space where $\Pi_{\text {ret }}^{\mu \nu}$ is given by Eq. (6). A pole of the retarded propagator, $\omega=E(\mathbf{p})-i \Gamma(\mathbf{p})$, contributes

$$
\int \frac{d \omega}{2 \pi i} \frac{e^{-i \omega t}}{\omega-E+i \Gamma}=e^{-i E t} e^{-\Gamma t}
$$

in the time domain. This shows that $\omega=E(\mathbf{p})$ is the dispersion relation of the excitation and $\Gamma(\mathbf{p})$ is the decay width.

Whenever the initial momentum distribution $f_{0}$ is anistropic, instabilities are present in the system. In $[12,17,18]$ it was shown that a new pole, $\omega=i \gamma$, appears in the retarded gluon propagator in the upper half complex plane. It corresponds to exponential growth $e^{\gamma t}$ in soft gluon density in the time domain. This happens as energy is transferred from quasiparticles to the soft chromomagnetic field as it deflects the quasiparticles which source an even stronger field [6]. This instability in soft gluon density has been studied extensively numerically; see [6] and references therein. The instability has furthermore been studied analytically in a longitudinally expanding, boost-invariant background; see, e.g., [19].

The presence of instabilities invalidates the assumption of a static medium. This can for instance be seen when evaluating photon production from the medium. At leading order in the strong coupling constant $g$, photons are produced through two distinct channels. The first channel is two-to-two scattering with a photon in the final stage, Fig. 1(a), which is unaffected by instabilities in gluon density since the mediator is a quark. ${ }^{4}$

\footnotetext{
${ }^{4}$ The interaction of quasiparticles includes two-to-two scattering such as in Fig. 1(b). This channel does not diverge in a static, nonequilibrium medium despite having a gluon mediator. This is because the mediator is a retarded propagator and not an $r r$ propagator. More physically, the gluon mediator is emitted by the quarks upon
}

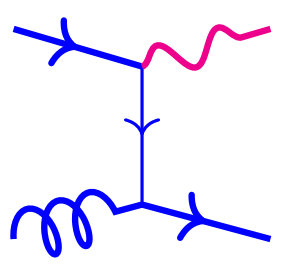

(a)

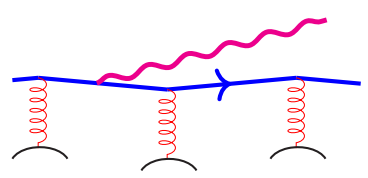

(c)

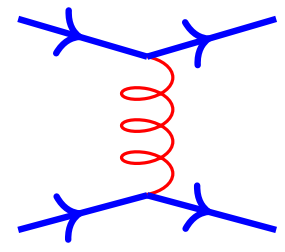

(b)

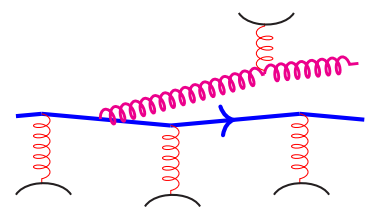

(d)
FIG. 1. Different processes in a weakly coupled quark-gluon plasma: (a) photon production through two-to-two scattering, (b) two-to-two scattering with gluon exchange, (c) photon emission triggered by in-medium interactions, and (d) gluon emission triggered by in-medium interactions.

Its rate has been calculated in a nonequilibrium plasma for various momentum distributions [20-22]. The second channel is medium-induced bremsstrahlung of a collinear photon; see Fig. 1(c). A quark is brought slightly off shell by kicks from the medium's soft gluons, which allows it to emit a photon. The probability for a kick to give the quark transverse momentum $\mathbf{q}_{\perp}$ is

$$
\mathcal{C}\left(\mathbf{q}_{\perp}\right)=g^{2} C_{F} \int \frac{d q_{0} d q_{z}}{(2 \pi)^{2}} 2 \pi \delta\left(q_{0}-q_{z}\right) \operatorname{Re} G_{r r}(Q)^{\mu \nu} \hat{K}_{\mu} \hat{K}_{v},
$$

where $\hat{K}^{\mu}=K^{\mu} / k$ is the direction of the quark $[12,13]$. Here the crucial ingredient is the correlator $G_{r r}^{\mu \nu}(x, y)=$ $\frac{1}{2}\left\langle\left\{A^{\mu}, A^{\nu}\right]\right\}$ which describes the density of soft excitations. In a static medium with initial time $t_{0}=-\infty$ it is given by

$$
G_{r r}(Q)=G_{\text {ret }} \Pi_{a a} G_{\mathrm{adv}}
$$

with $G_{\text {adv }}=G_{\text {ret }}^{*}$ and $\Pi_{a a}$ denotes the probability to create the excitation. During emission of a collinear photon, the quark can receive arbitrarily many kicks from the soft gluons. The kicks act coherently and tend to reduce the rate of emission; this is the Landau-Pomeranchuk-Migdal effect $[14,23,24]$. Thus in a static medium the rate of photon production through bremsstrahlung has a complicated dependence on $\mathcal{C}\left(\mathbf{q}_{\perp}\right)$ which can be seen in Eqs. (23) and (24) [13].

We can now finally see how instabilities invalidate the assumption of a static medium when calculating photon production through medium-induced bremsstrahlung. Roughly speaking the $r r$ propagator for the instability mode can be approximated as

$$
G_{r r} \sim \frac{1}{\left(q^{0}-i \gamma\right)\left(q^{0}+i \gamma\right)}
$$

interaction and thus does not depend on the accumulated density of gluons in the system. 
Substituting this into Eq. (9) and ignoring $q_{z}$ dependence gives

$$
\mathcal{C}\left(\mathbf{q}_{\perp}\right) \sim \frac{1}{2 \gamma\left(\mathbf{q}_{\perp}\right)} .
$$

We thus see that for slowly growing modes $\gamma \rightarrow 0$ at finite $\mathbf{q}_{\perp}$ the probability for interacting with soft gluons diverges. This is a sign that our handling of instabilities in a static medium is incorrect.

The function $\mathcal{C}\left(\mathbf{q}_{\perp}\right)$ in Eq. (9) not only appears in photon production but also when calculating the rate of jet-medium interaction [23,25], as well as interaction of quasiparticles [12]. All of these processes thus suffer from the same divergence in a naive calculation in a static medium. Furthermore, a similar problem arises when calculating the imaginary part of the heavy quark potential in a nonequilibrium medium [11].

\section{OVERVIEW OF RESULTS AND IMPLICATIONS FOR PHENOMENOLOGY}

We must go beyond the assumption of a static medium to calculate photon production through bremsstrahlung in a nonequilibrium QGP as is found in the hydrodynamic stage of heavy-ion collisions. Otherwise, we get nonsensical results because of instabilities in soft gluon density. However, including the time evolution of the medium in general is a complicated task, especially since we can no longer do Fourier transforms which are essential to get simple equations for the Landau-Pomeranchuk-Migdal effect. To be able to handle this task, we consider the simplest setup imaginable for the medium and draw lessons from it for more realistic settings. This furthermore gives a rare opportunity to do analytic calculation in nonequilibrium plasma.

In our setup the plasma is initially composed of hard quasiparticles with energy $\Lambda$ while soft gluons with energy $g \Lambda$ are absent. The initial condition at $t_{0}=0$ is given by a slightly anisotropic quasiparticle distribution $f_{0}(\mathbf{p})$. The anisotropy is defined by

$$
\xi \sim \frac{\left|\left\langle p_{z}\right\rangle-\left\langle p_{\perp}\right\rangle\right|}{\left\langle p_{z}\right\rangle}
$$

where $\left\langle p_{z}\right\rangle$ and $\left\langle p_{\perp}\right\rangle$ are the momentum distribution's typical momenta. We assume that $\xi \ll g$. This guarantees that the growth of instabilities is slow enough for us to have a handle on the calculation. The small anisotropy thus allows for a controlled calculation. For higher values of anisotropy, such as in phenomenological applications [26], the calculation could be extended beyond this controlled regime, much like when perturbative calculations are extended to realistic coupling strength. We furthermore only consider times shortly after the starting time. This ensures that the density of the soft gluons does not become so high that the HTL approximation is invalidated.

In Sec. IV we calculate the propagators that describe soft gluons in this setup. The retarded correlator becomes

$$
G_{\text {ret }}\left(t_{x}, t_{y} ; \mathbf{p}\right)=\int_{\alpha} \frac{d p^{0}}{2 \pi} e^{-i p^{0}\left(t_{x}-t_{y}\right)} G_{\text {ret }}\left(p^{0}, \mathbf{p}\right) .
$$

The propagator is written in the time domain where $t_{x}, t_{y}>0$ are the times of the two fields. Since we assume an infi-

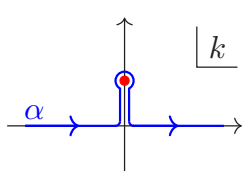

(a)

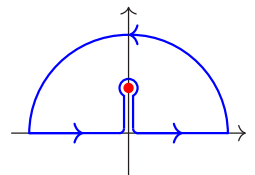

(b)

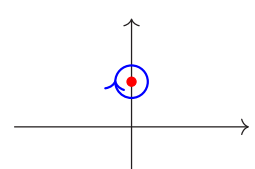

(c)
FIG. 2. Integration contours in the frequency domain. (a) The contour $\alpha$ runs along the real axis and goes above all poles in the upper half plane. (b) The contour $\alpha$ continued in the upper half plane. (c) The contour $\gamma$ circles all poles in the upper half plane.

nite spatial extension we can define a three-momentum $\mathbf{p}$ by Fourier transform. The function

$$
G_{\text {ret }}\left(p^{0}, \mathbf{p}\right)=\left[\left(G_{\text {ret }}^{0}(P)\right)^{-1}-\Pi_{\text {ret }}(P)\right]^{-1}
$$

is the same as in Eq. (7). It generally has poles in the upper half complex plane which correspond to instabilities [17]. Crucially, we must choose a contour $\alpha$ that goes above all poles in the upper half complex plane, as in Fig. 2(a). An instability pole $p^{0}=i \gamma$ then gives $G_{\mathrm{ret}} \sim e^{\gamma\left(t_{x}-t_{y}\right)}$ for $t_{x}>t_{y}$ which grows exponentially, showing that the system is unstable to perturbations. Choosing the contour in this way also guarantees that $G_{\text {ret }}\left(t_{x}, t_{y} ; \mathbf{k}\right)=0$ for $t_{y}>t_{x}$.

The important ingredient when calculating photon emission is the $r r$ correlator of soft gluons which describes the soft gluon density. To find an expression for it we must separate between two scales, namely, the soft scale $g \Lambda$ and the instability growth rate $\gamma \sim \xi g \Lambda .5^{5}$ As an example we write the retarded correlator in Eq. (15) as

$$
G_{\text {ret }}(K)=\widehat{G}_{\text {ret }}(K)+\sum_{i} \frac{A_{i}}{k^{0}-i \gamma_{i}}
$$

where $\widehat{G}_{\text {ret }}$ only has poles and branch cuts of order $g \Lambda$ while $\gamma_{i}$ are all poles of order $\xi g \Lambda$, including instability poles. Using a number of controlled approximations, explained in Sec. IV, we then get the $r r$ correlator at early times when the gluon occupation density is not so high that the HTL approximation is invalidated. It is

$$
\begin{aligned}
G_{r r}\left(t_{x}, t_{y} ; \mathbf{k}\right) \approx & \int \frac{d k^{0}}{2 \pi} e^{-i k^{0}\left(t_{x}-t_{y}\right)} \widehat{G}_{r r}(K) \\
& +\sum_{i, j} \frac{A_{i} \Pi_{a a}(0) A_{j}^{*}}{\gamma_{i}+\gamma_{j}}\left[e^{\left(\gamma_{i}+\gamma_{j}\right) T}-1\right]
\end{aligned}
$$

with

$$
\widehat{G}_{r r}(K)=\widehat{G}_{\text {ret }}(K) \Pi_{a a}(K) \widehat{G}_{\text {adv }}(K)
$$

and $\widehat{G}_{\text {adv }}=\widehat{G}_{\text {ret }}^{*}$. Here $T=\frac{t_{x}+t_{y}}{2}$ is the time that has passed since the system was initialized.

The $r r$ propagator in Eq. (17) has a clear physical interpretation. The first term has no information about the initial time. It is of the same form as the $r r$ correlator in a static medium,

${ }^{5}$ Strictly speaking, $\gamma \sim \xi^{3 / 2}$ but we will not need these more precise estimates in our paper [27]. 
Eq. (9), except that instability modes are not included. The second term describes the instability modes and shows exponential growth at scale $\gamma \sim \xi g \Lambda$. It vanishes at the initial time $T=0$ when the instability modes are not occupied.

Most importantly, Eq. (17) is finite for slow growth rate $\gamma \rightarrow 0$. In particular, the second term in the square bracket, which comes from the initial condition, cancels the divergence of $e^{\gamma_{i}} e^{\gamma_{j}} /\left(\gamma_{i}+\gamma_{j}\right)$. This shows that including the time evolution of the instabilities cures the spurious divergences we encountered in Eq. (12), giving a finite collision kernel $\mathcal{C}\left(\mathbf{q}_{\perp}\right)$.

It is instructive to evaluate the collision kernel $\mathcal{C}\left(\mathbf{q}_{\perp}\right)$ in our particular setup of small anisotropy. This kernel describes the probability for a quark traveling at the speed of light to interact with a single gluon. Assuming for simplicity that there is only one instability mode with growth rate $\gamma$, and Fourier transforming Eq. (17), we can write

$$
G_{r r}(K)=\widehat{G}_{r r}(K)+\frac{A \Pi_{a a}(0) A^{*}}{2 \gamma}\left[e^{2 \gamma T}-1\right] 2 \pi \delta\left(k^{0}\right) .
$$

Substituting into Eq. (9) then gives the collision kernel

$$
\mathcal{C}\left(\mathbf{q}_{\perp}\right)=\widehat{\mathcal{C}}\left(\mathbf{q}_{\perp}\right)+\left.g^{2} C_{F} \widehat{K}_{\mu} \widehat{K}^{\nu}\left[A \Pi_{a a}(0) A^{*}\right]^{\mu \nu} \frac{e^{2 \gamma T}-1}{2 \gamma}\right|_{q_{z}=0}
$$

where $\gamma=\gamma(\mathbf{q})$. The first term is independent of instability modes and is given by

$$
\widehat{\mathcal{C}}\left(\mathbf{q}_{\perp}\right)=g^{2} C_{F} \int \frac{d q_{0} d q_{z}}{(2 \pi)^{2}} 2 \pi \delta\left(q_{0}-q_{z}\right) \operatorname{Re} \widehat{G}_{r r}(Q)^{\mu \nu} \hat{K}_{\mu} \hat{K}_{\nu} .
$$

It describes a fluctuating cloud of soft gluons that are sourced by hard quasiparticles at each instant. The effect of the soft gluon cloud on photon production thus only depends on the instantaneous, macroscopic properties of the medium. It contains a wealth of nonequilibrium information coming from the nonequilibrium distribution of hard quasiparticles. The second term in Eq. (20) depends on the occupation density of the instabilities. It is time dependent and thus depends on the whole history of the medium. As we have argued it is finite as $\gamma \longrightarrow 0$, showing explicitly that the collision kernel is finite.

We derived Eq. (20) rigorously for a particular setup where a system is started with a small anisotropy, $\xi \ll g$. Furthermore, we follow the system shortly after the initialization so that the HTL approximation remains valid. At later times and in a more general setup the instability modes should be described by some general correlator $G_{r r \text { inst }}^{\mu \nu}\left(t_{x}, t_{y} ; \mathbf{k}\right)$ that quantifies their occupancy and must be calculated numerically. Assuming that the correlator does not change substantially during the time it takes to emit a photon, $t_{x}-t_{y} \sim 1 / g^{2} \Lambda$, this suggests a collision kernel

$$
\mathcal{C}\left(\mathbf{q}_{\perp}\right)=\widehat{\mathcal{C}}\left(\mathbf{q}_{\perp}\right)+\left.g^{2} C_{F} \widehat{K}_{\mu} \widehat{K}^{\nu} G_{r r, \text { inst }}^{\mu \nu}(T, T ; \mathbf{k})\right|_{q_{z}=0}
$$

where $T$ is the time at which a photon is emitted.

In both Eqs. (20) and (22) we have two scales: First, there is a fluctuating gluon cloud at energy $g \Lambda$. An emitting quark gets kicks from the cloud which take time $1 / g \Lambda$ and which

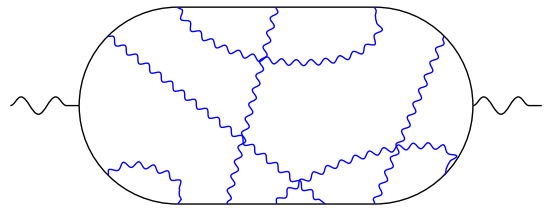

FIG. 3. An example of diagrams that are summed up to evaluate photon production in an Abelian background field. The thin lines joining quark propagators are background field insertions.

are ordered in time as the time between two kicks is typically $1 / g^{2} \Lambda$. This makes it possible to sum up all the momentum kicks as must be done at leading order in perturbation theory. Second, there are the instability fields the wavelength of which is longer than the time it takes to emit a photon. Therefore, they are not ordered in time, forcing one to evaluate diagrams like Fig. 3 for photon emission in a background field. This is difficult in general. However, in Secs. V and VI we take a modest step in that direction by showing that in an Abelian plasma the long-wavelength instability field does not affect photon emission at leading and next-to-leading order in $l \Delta t \ll 1$, irrespective of occupation density, where $l$ is a typical transverse kick of instability modes and $\Delta t$ is the time needed for photon emission.

For phenomenological purposes worries about including the effect of long-wavelength instability fields are likely superfluous. First, we have shown that there are no spurious divergences when time dependence is taken into account, meaning that instabilities are not enhanced through divergences. Second, the contribution of instabilities to the collision kernel will always depend on their occupation density, like in Eq. (20). Thus, the instability contribution can be dropped if they have a low enough occupation density. This is borne out by detailed classical-statistical simulations which suggest that plasma instabilities only play a role in the very early stages of heavy-ion collisions $[28,29]$. These calculations describe a weakly coupled, highly occupied classical system with fluctuating initial conditions coming from the color-glass condensate. There the instabilities are important in the approach to a universal, nonequilibrium attractor but once the attractor is reached detailed information on the initial stages is forgotten and the dynamics is dominated by a turbulent cascade towards higher energies until thermalization is reached.

For the phenomenology of photon production in a nonequilibrium QGP, it is therefore reasonable to neglect the contribution of instabilities and use the function $\widehat{\mathcal{C}}$ in Eq. (21). This function is nontrivial and has not yet been calculated fully for a given nonequilibrium distribution. Using it guarantees a finite rate which still includes the essential nonequilibrium information, both from the nonequilibrium quasiparticle distribution $f$ as well as from the soft gluon cloud sourced at each instant by the quasiparticles. The same procedure works for medium-induced jet splitting which also depends on the function $\widehat{\mathcal{C}}$. We will report on photon production in a nonequilibrium QGP, using this procedure [30,31]. 
Applying this prescription, the rate of emitting photons with momentum $\mathbf{k}$ through bremsstrahlung is

$$
\begin{aligned}
k \frac{d R}{d^{3} k}= & \frac{3 Q^{2} \alpha_{E M}}{4 \pi^{2}} \int \frac{d^{3} p}{(2 \pi)^{3}} F(P+K)[1-F(P)] \\
& \times \frac{p^{z 2}+\left(p^{z}+k\right)^{2}}{2 p^{z 2}\left(p^{z}+k\right)^{2}} \mathbf{p}_{\perp} \cdot \operatorname{Re} \mathbf{f}(\mathbf{p} ; \mathbf{k})
\end{aligned}
$$

when the photon is emitted in the $z$ direction [13]. Here $\operatorname{Re} \mathbf{f}(\mathbf{p})$ can be thought of as the probability for the quark to gain transverse momentum $\mathbf{p}$ because of medium kicks. It is solved by the Boltzmann-like equation

$$
\mathbf{p}_{\perp}=i \delta E \mathbf{f}\left(\mathbf{p}_{\perp}\right)+\int \frac{d^{2} q_{\perp}}{(2 \pi)^{2}} \widehat{\mathcal{C}}\left(\mathbf{q}_{\perp}\right)\left[\mathbf{f}\left(\mathbf{p}_{\perp}\right)-\mathbf{f}\left(\mathbf{p}_{\perp}+\mathbf{q}_{\perp}\right)\right],
$$

where $\widehat{\mathcal{C}}$ comes from Eq. (21).

Our rigorous derivation of Eqs. (17) and (20) assumed a small anisotropy $\xi \ll g$. At higher anisotropy, the gluon occupation density grows rapidly and the HTL approximation is violated before the medium has had time to emit a photon. More generally, our discussion has assumed that the instability modes change slowly during photon emission; see Eq. (22). At higher anisotropy the occupation density of the instability modes changes rapidly while a photon is being emitted. Thus we need a collision kernel in the time domain that changes with each gluon kick from the instability modes. Including such a collision kernel changes the structure of the equations describing photon production through bremsstrahlung and is beyond the scope of this paper. Nevertheless, our calculation shows that even at this higher anisotropy the new collision kernel will have no divergences due to instabilities. We know this because we can still derive the effect of the instabilities at higher anisotropy at very early times before the HTL approximation breaks down. This calculation which is discussed further at the end of Sec. IV, shows that spurious divergences in the $r r$ correlator only arise because time dependence is ignored, even at higher anisotropy.

\section{CORRELATORS FOR UNSTABLE FIELDS}

We now turn to derive the correlators in Eqs. (14) and (17) for an anisotropic plasma shortly after specifying the initial condition in our setup. We assume a small anisotropy $\xi \ll g$. The retarded propagator is defined by

$$
\begin{aligned}
G_{\text {ret }}(x, y)=G_{\text {ret }}^{0}(x, y)+ & \int d^{4} z \int d^{4} w G_{\text {ret }}^{0}(x, z) \\
& \times \Pi_{\text {ret }}(z, w) G_{\text {ret }}(w, y)
\end{aligned}
$$

where $\Pi_{\text {ret }}$ is the retarded self-energy and $G_{\text {ret }}^{0}$ is the bare retarded propagator (see, e.g., [32] and Sec. 3 of [33]). In a static system, such as thermal equilibrium, this equation can be solved by Fourier transforming to the frequency domain, thanks to translational invariance which guarantees that $G_{\text {ret }}(x, y)=G_{\text {ret }}(x-y)$. We must take a different route to solve Eq. (25) since time translational invariance is broken by instabilities. We assume that our system has infinite spatial extension so that the spatial dependence can be described in Fourier space.
We start our system at initial time $t_{0}=0$. The time integrals in Eq. (25) range over all times greater than the initial time. Using the properties of retarded functions, we write ${ }^{6}$

$$
\begin{aligned}
G_{\mathrm{ret}}(x, y)= & G_{\mathrm{ret}}^{0}(x-y) \\
& +\int_{y}^{x} d z \int_{y}^{z} d w G_{\mathrm{ret}}^{0}(x-z) \\
& \times \Pi_{\mathrm{ret}}(z-w) G_{\mathrm{ret}}(w, y),
\end{aligned}
$$

where the dependence on three-momentum is omitted. We have $\Pi_{\text {ret }}(z, w)=\Pi_{\text {ret }}(z-w)$ in the HTL approximation, valid at the early times we consider when the soft gluon density is not too high. This equation has the same form as in equilibrium because the initial time does not appear explicitly. Furthermore, $G_{\text {ret }}(x+\tau, y+\tau)$ is a solution of Eq. (26) for any $\tau$. This suggests that we can write $G_{\text {ret }}(x, y)=$ $G_{\text {ret }}(x-y)$. We will therefore try to find a solution ${ }^{7}$

$$
G_{\text {ret }}(x, y)=\int_{\alpha} \frac{d k}{2 \pi} e^{-i k(x-y)} G_{\text {ret }}(k)
$$

for some function $G_{\text {ret }}(k)$. It is enough to find one such solution because the solution of Eq. (26) is unique. The contour $\alpha$ goes along the real line and above all instability poles that $G_{\text {ret }}(k)$ might have in the upper half complex plane; see Fig. 2(a). This ensures that $G_{\text {ret }}(x, y)=0$ for $y>x$.

We will now evaluate the last term in Eq. (26) in detail. Substituting Eq. (27) and the Fourier transforms ${ }^{8}$ of $G_{\text {ret }}^{0}$ and $\Pi_{\text {ret }}$ we write that term as

$$
\begin{aligned}
& \int_{y}^{x} d z \int_{y}^{z} d w \int \frac{d k_{1}}{2 \pi} \int \frac{d k_{2}}{2 \pi} \int_{\alpha} \frac{d k_{3}}{2 \pi} \\
& \quad \times e^{-i k_{1}(x-z)} e^{-i k_{2}(z-w)} e^{-i k_{3}(w-y)} G_{\mathrm{ret}}^{0}\left(k_{1}\right) \Pi_{\mathrm{ret}}\left(k_{2}\right) G_{\mathrm{ret}}\left(k_{3}\right) .
\end{aligned}
$$

The time integrals can be done explicitly. This would not be possible if the time integrals were written for all $z, w \geqslant 0$ since the integral with $e^{-i k_{3}(w-y)}$ would not converge with $k_{3}$ in the upper half complex plane. In the end we get

$$
\int \frac{d k_{1}}{2 \pi} \int \frac{d k_{2}}{2 \pi} \int_{\alpha} \frac{d k_{3}}{2 \pi} G_{\text {ret }}^{0}\left(k_{1}\right) \Pi_{\text {ret }}\left(k_{2}\right) G_{\text {ret }}\left(k_{3}\right) f\left(k_{1}, k_{2}, k_{3}\right)
$$

\footnotetext{
${ }^{6}$ To avoid clutter we denote time coordinates with $x, y, z, w$ instead of $x^{0}, y^{0}, z^{0}, w^{0}$.

${ }^{7}$ Here $k$ can be seen as a frequency coordinate. We will write $k$ instead of $k^{0}$.

${ }^{8}$ Since $\Pi_{\text {ret }}(x, y)=\Pi_{\text {ret }}(x-y)$, we can define a Fourier transform in the usual way which justifies integrating $k_{2}$ over the real line in Eq. (28). For the full retarded function, a Fourier transform $G_{\text {ret }}(k)=$ $\int d(x-y) e^{i k(x-y)} G_{\text {ret }}(x-y)$ is ill defined since $G_{\text {ret }}(x-y)$ has an exponentially growing instability part. Formally, we could define a Laplace transform with the inverse given by Eq. (27). However, we prefer avoiding formal integrals which do not converge. In the end, the time domain is the only physical domain in a nonequilibrium system and $G_{\text {ret }}(k)$ is just some function that gives the correct retarded function when substituted in Eq. (27).
} 
where

$$
f\left(k_{1}, k_{2}, k_{3}\right)=-\frac{e^{-i k_{1}(x-y)}}{\left(k_{1}-k_{2}\right)\left(k_{1}-k_{3}\right)}-\frac{e^{-i k_{2}(x-y)}}{\left(k_{2}-k_{1}\right)\left(k_{2}-k_{3}\right)}-\frac{e^{-i k_{3}(x-y)}}{\left(k_{3}-k_{1}\right)\left(k_{3}-k_{2}\right)} .
$$

Some tricks are needed to evaluate Eq. (29). We notice that the function $f\left(k_{1}, k_{2}, k_{3}\right)$ has no poles in its variables. Thus, we can include a principal value for each term in the function by substituting

$$
f\left(k_{1}, k_{2}, k_{3}\right) \longrightarrow \frac{1}{8} \sum_{\substack{\left\{k_{1} \rightarrow k_{1}+i \epsilon_{1}\right\} \\\left\{k_{1} \rightarrow k_{1}-i \epsilon_{1}\right\}}} \sum_{\substack{\left\{k_{2} \rightarrow k_{2}+i \epsilon_{2}\right\} \\\left\{k_{2} \rightarrow k_{2}-i \epsilon_{2}\right\}}} \sum_{\substack{\left\{k_{3} \rightarrow k_{3}+i \epsilon_{3}\right\} \\\left\{k_{3} \rightarrow k_{3}-i \epsilon_{3}\right\}}} f\left(k_{1}, k_{2}, k_{3}\right) .
$$

Here $\epsilon_{1}, \epsilon_{2}, \epsilon_{3}>0$ are set to zero in the end. The result must be independent of the order in which they are set to zero. As is shown in Appendix A we can then evaluate the momentum integrals in Eq. (29) using the residue theorem. Doing so requires continuing the integration contours to the correct half plane which only contains poles of the function $f$. The final result is

$$
\int_{\alpha} \frac{d k}{2 \pi} e^{-i k(x-y)}\left[G_{\mathrm{ret}}(k)-G_{\mathrm{ret}}^{0}(k)-G_{\mathrm{ret}}^{0}(k) \Pi_{\mathrm{ret}}(k) G_{\mathrm{ret}}(k)\right]=0 .
$$

From this we immediately see that

$$
G_{\text {ret }}\left(k^{0}, \mathbf{k}\right)=\left\{\left[G_{\text {ret }}^{0}(k)\right]^{-1}-\Pi_{\text {ret }}(k)\right\}^{-1},
$$

confirming our expression for $G_{\text {ret }}$ in Eq. (14). We note that the advanced correlator can easily be shown to be

$$
G_{\mathrm{adv}}(x, y)=\int_{\widetilde{\alpha}} \frac{d k}{2 \pi} e^{-i k(x-y)} G_{\mathrm{adv}}(k),
$$

where $G_{\text {adv }}(k)=G_{\text {ret }}(k)^{*}$ and the integration contour is $\widetilde{\alpha}=\tilde{\alpha}^{*}$ which goes below all poles of $G_{\text {adv }}(k)$.

We have found the retarded and advanced correlators. The other two-point correlator is $G_{r r}=\frac{1}{2}\left\langle\left\{A^{\mu}(x), A^{\nu}(y)\right\}\right\rangle$ which gives the occupation density of gluonic modes in the medium. It is

$$
G_{r r}(x, y)=\int_{0}^{x} d z \int_{0}^{y} d w G_{\mathrm{ret}}(x-z) \Pi_{a a}(z-w) G_{\mathrm{adv}}(w-y)
$$

where the integration limits have been rewritten using properties of the retarded and advanced functions, as well as the initial time $t_{0}=0$ [33]. In general there is an additional term corresponding to correlation with the initial state. Assuming that there are no soft gluons in the initial state, we can omit that term but it could easily be included in our calculations. The integrals depend explicitly on the initial time so we expect that $G_{r r}(x, y) \neq G_{r r}(x-y)$. Substituting the Fourier transform of the HTL $\Pi_{a a}$ as well as Eqs. (27) and (34) gives

$$
\begin{aligned}
G_{r r}(x, y)= & \int_{\alpha} \frac{d k_{1}}{2 \pi} \int \frac{d k_{2}}{2 \pi} \int_{\widetilde{\alpha}} \frac{d k_{3}}{2 \pi}\left[-e^{-i k_{2} x} e^{i k_{2} y}+e^{-i k_{1} x} e^{i k_{2} y}+e^{-i k_{2} x} e^{i k_{3} y}-e^{-i k_{1} x} e^{i k_{3} y}\right] \\
& \times \frac{1}{\left(k_{1}-k_{2}\right)\left(k_{2}-k_{3}\right)} G_{\mathrm{ret}}\left(k_{1}\right) \Pi_{a a}\left(k_{2}\right) G_{\mathrm{adv}}\left(k_{3}\right)
\end{aligned}
$$

after doing the time integrals.

In order to evaluate the remaining integrals in Eq. (36) we must think about the scales of the problem. The retarded correlator is at two momentum scales:

$$
G_{\mathrm{ret}}(k)=\widehat{G}_{\mathrm{ret}}(k)+\sum_{i} \frac{A_{i}}{k-i \gamma_{i}} .
$$

Here, $\widehat{G}_{\text {ret }}$ only has poles and branch cuts of order $g \Lambda$ which are all in the lower half complex plane while $\gamma_{i}$ are all poles of order $\xi g \Lambda$, with $\xi \ll g$ the initial anisotropy of the system. We have split $G_{\text {ret }}$ into a fluctuating part $\widehat{G}_{\text {ret }}$ that is continually sourced by quasiparticles and an instability part that includes time evolution. Similarly, we write

$$
G_{\mathrm{adv}}(k)=\widehat{G}_{\mathrm{adv}}(k)+\sum_{j} \frac{A_{j}^{*}}{k+i \gamma_{j}}
$$

where $\widehat{G}_{\text {adv }}=\widehat{G}_{\text {ret }}^{*}$. The self-energy $\Pi_{a a}$ only has poles and branch cuts of order $g \Lambda$.

We need to be careful when writing the retarded correlator as in Eq. (37). The correlator has a branch cut from $\omega=$ $-|\mathbf{k}|-i \epsilon$ to $|\mathbf{k}|-i \epsilon$ which corresponds to Landau damping. The branch cut is most often chosen to lie just below the real axis but then it will be partially at the scale $\xi g \Lambda$ which spoils the separation of scales in Eq. (37). The remedy is to 


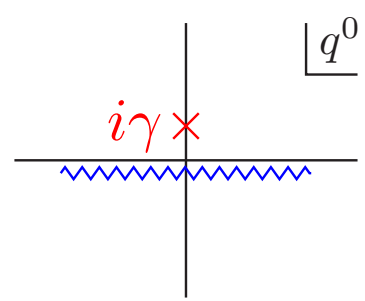

(a)

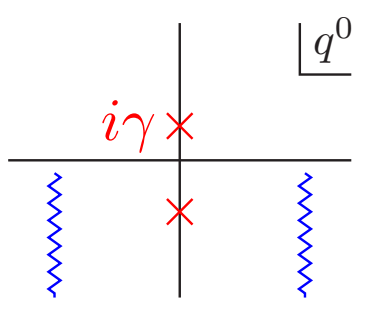

(b)
FIG. 4. Different branch cuts for Landau damping in the retarded gluon correlator: (a) the typical branch cut which spoils a separation of scales and (b) an alternative branch cut which respects separation of scales; if it is chosen additional poles appear in the second Riemann sheet.

choose a branch cut that avoids the $\xi g \Lambda$ region; see Fig. 4 . This results in new decaying modes on the second Riemann sheet [34]. Ultimately, the retarded propagator only exists in the time domain where it is independent of the branch cut we choose.

We will use controlled approximations to evaluate the $r r$ correlator in Eq. (36). First, we assume that $x, y \gg 1 / g^{2} \Lambda$ so that sufficient time has passed since the system was initialized. This allows us to drop any term with $e^{-i a x}$ where $\operatorname{Im} a<0$ and $\operatorname{Im} a \sim g \Lambda$, as correlations with the initial condition are damped when sufficient time has passed. Second, we can assume that $x-y \sim 1 / g^{2} \Lambda$ since this is the time that medium-induced emission takes. This allows us to drop any term with $e^{-i a x}$ where $\operatorname{Re} a \sim g \Lambda$, as it oscillates very rapidly during emission and cancels out. The terms we drop would also be present in a thermally equilibrated system started at an initial time $t_{0}=0$. They tell us little about the nonequilibrium physics we are interested in.

These approximations allow us to to evaluate the $r r$ propagator at early times. Using the same calculational tricks as before, a lengthy calculation given in Appendix A shows that

$$
\begin{aligned}
G_{r r}(x, y) \approx & \int \frac{d k}{2 \pi} \widehat{G}_{\mathrm{ret}}(k) \Pi_{a a}(k) \widehat{G}_{\mathrm{adv}}(k) e^{-i k(x-y)}+\sum_{i} \int \frac{d k}{2 \pi} \frac{A_{i}}{k-i \gamma_{i}} \Pi_{a a}(k) \widehat{G}_{\mathrm{adv}}(k)\left(e^{-i k x}-e^{\gamma_{i} x}\right) e^{i k y} \\
& +\sum_{j} \int \frac{d k}{2 \pi} \widehat{G}_{\mathrm{ret}}(k) \Pi_{a a}(k) \frac{A_{j}^{*}}{k+i \gamma_{j}} e^{-i k x}\left(e^{i k y}-e^{\gamma_{j} y}\right)+\sum_{i, j} \int \frac{d k}{2 \pi} \frac{A_{i}}{k-i \gamma_{i}} \Pi_{a a}(k) \frac{A_{j}^{*}}{k+i \gamma_{j}} \\
& \times\left(e^{-i k x}-e^{\gamma_{i} x}\right)\left(e^{i k y}-e^{\gamma_{j} y}\right),
\end{aligned}
$$

where the terms correspond to fluctuating contributions $k \sim$ $g \Lambda$, instability contributions $k \sim \xi g \Lambda$, or their cross terms.

Equation (39) has a simple interpretation. Schematically, a mode $e^{-i E t-\gamma t}$ of the retarded function contributes

$$
\int_{-\infty}^{x} d t e^{i k^{0} t} e^{-i E t-\gamma t}=\frac{-i}{k^{0}-E+i \gamma} e^{i\left(k^{0}-E+i \gamma\right) x}
$$

to the $r r$ correlator in a system in thermal equilibrium with initial condition at $t \rightarrow-\infty$. This expression has a pole at $k^{0}=E-i \gamma$. However, in a nonequilibrium system with initial time at $t=0$ the corresponding integral is

$$
\int_{0}^{x} d t e^{i k^{0} t} e^{-i E t-\gamma t}=\frac{-i}{k^{0}-E+i \gamma}\left[e^{i\left(k^{0}-E+i \gamma\right) x}-1\right],
$$

which has no pole. In a similar fashion, there should strictly speaking be no poles in Eq. (39): for a pole $b \sim g \Lambda$ of $\widehat{G}_{\text {ret }}$ we should have

$$
\frac{1}{k-b}\left(e^{-i k x}-e^{-i b x}\right)
$$

Nevertheless, in using our approximations we have dropped all terms $\approx e^{-i b x}$ since sufficient time has passed to eliminate all traces of an initial time $t_{0}=0$. Conversely, we must retain the analogous factors $e^{\gamma x}$ for instability modes since they grow exponentially in time.

It is instructive to rewrite Eq. (39). We can drop cross-terms between instability and fluctuating modes since the decay or oscillations of fluctuating modes dominates over the slow growth rate of instability terms. ${ }^{9}$ The last term in Eq. (39) has no poles because of the exponentials and can thus be written with a different contour:

$$
\begin{aligned}
& \sum_{i, j} \int_{\beta} \frac{d k}{2 \pi} \frac{A_{i}}{k-i \gamma_{i}} \Pi_{a a}(k) \frac{A_{j}^{*}}{k+\gamma_{j}} \\
& \quad \times\left(e^{-i k x}-e^{\gamma_{i} x}\right)\left(e^{i k y}-e^{\gamma_{j} y}\right) .
\end{aligned}
$$

Here $\beta$ is a contour that goes along the real line and above all instability poles of $G_{\text {ret }}$ in the upper half plane and below all instability poles of $G_{\text {adv }}$ in the lower half plane; see Fig. 5 . Doing the contour integrals then gives

$$
\frac{A_{i} \Pi_{a a}(0) A_{j}^{*}}{\gamma_{i}+\gamma_{j}}\left[e^{\gamma_{i} x} e^{\gamma_{j} y}-\theta(x-y) e^{\gamma_{i}(x-y)}-\theta(y-x) e^{\gamma_{j}(y-x)}\right]
$$

\footnotetext{
${ }^{9}$ This can be seen in a simple way. Let us consider a term $e^{(i d+c) t}$ where $d \sim g \Lambda$ gives oscillations and $c \sim \xi g \Lambda$ gives exponential growth. Averaging over the time of interaction in medium-induced emission corresponds to introducing an initial time $t_{0}$ which varies over scale $1 / g^{2} \Lambda$. This can, e.g., be done by integrating $e^{(i d+c)\left(t-t_{0}\right)} e^{-t_{0}^{2} / 2 \sigma^{2}}$ over the initial time $t_{0}$ where the Gaussian with width $\sigma \sim 1 / g^{2} \Lambda$ corresponds to averaging the time of emission over the time a typical emission takes. Integrating over $t_{0}$ then gives a factor $e^{-\frac{1}{2} \sigma^{2}\left(d^{2}-c^{2}+2 i c d\right)}$ which is heavily suppressed since $d \gg c$ and $\sigma d \gg 1$. A full field theoretical calculation gives the same exponential suppression.
} 


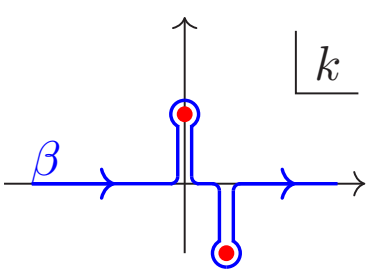

FIG. 5. The contour $\beta$ goes above instability poles of $G_{\text {ret }}$ in the upper half plane and below instability poles of $G_{\text {adv }}$ in the lower half plane. Otherwise, it goes along the real axis.

where we can ignore poles ${ }^{10}$ of $\Pi_{a a}(k)$ and write $\Pi_{a a}\left(a_{i}\right) \approx$ $\Pi_{a a}\left(a_{j}^{*}\right) \approx \Pi_{a a}(0)$. Using that $a(x-y) \sim \xi / g \ll 1$ gives our final expression for the full $r r$ correlator which reproduces Eq. (17):

$$
\begin{aligned}
G_{r r}\left(t_{x}, t_{y} ; \mathbf{k}\right) \approx & \int \frac{d k^{0}}{2 \pi} e^{-i k^{0}\left(t_{x}-t_{y}\right)} \\
& \times \widehat{G}_{\mathrm{ret}}\left(k^{0} ; \mathbf{k}\right) \Pi_{a a}\left(k^{0} ; \mathbf{k}\right) \widehat{G}_{\mathrm{adv}}\left(k^{0} ; \mathbf{k}\right) \\
& +\sum_{i, j} \frac{A_{i} \Pi_{a a}(0) A_{j}^{*}}{\gamma_{i}+\gamma_{j}}\left[e^{\left(\gamma_{i}+\gamma_{j}\right) T}-1\right]
\end{aligned}
$$

where $\gamma_{i}$ and $A_{i}$ are functions of the three-momentum $\mathbf{k}$, and $T=\frac{t_{x}+t_{y}}{2}$ is the time that has passed since the system was initialized.

Recall that this calculation has assumed a small anisotropy, $\xi \ll g$. At higher anisotropy the HTL approximation breaks down before the medium has had time to emit a photon. Nevertheless, much of the derivation of the $r r$ correlator is valid for higher values of anisotropy in the small time window after initialization during which the HTL approximation is still valid. In particular, Eq. (39) remains valid, except that decaying and oscillating modes at energy $g \Lambda$ cannot be dropped. (Their inclusion shortly after initialization of a system is discussed in [35].) For our purposes we only need the last term in Eq. (39) which describes the instability modes and has no decaying or oscillating modes. This is the term that, when treated incorrectly, leads to spurious divergences in a collision kernel. Just like at lower anisotropy it can be rewritten to give Eq. (44). We see this expression is finite when $\gamma=\gamma_{i}=\gamma_{j} \longrightarrow 0$, precisely because of the last two terms which come from the initial condition. This shows that spurious divergences in the $r r$ correlator, and by extension the collision kernel, only arise because time dependence is ignored. As explained in Sec. III a full calculation of the collision kernel at higher anisotropy is involved and beyond the scope of this paper. But importantly, we have shown that it will not suffer from spurious divergences.

\footnotetext{
${ }^{10}$ The fact that poles of $\Pi_{a a}$ can be ignored can be seen as follows: Let us write $\Pi_{a a}$ as $A /(k-B)$ where $B \sim g \Lambda$ is a pole and $A$ is the residue. Upon performing the contour integral, the pole $B$ will contribute $A /\left(B-a_{i}\right)\left(B-a_{j}^{*}\right) \sim A /\left(g^{2} \Lambda\right)$ while an instability pole will contribute $A /\left(a_{i}-B\right)\left(a_{i}-a_{j}^{*}\right) \sim A / \xi g^{2} \Lambda$ which is much bigger.
}

\section{QUARK PROPAGATORS IN LONG-WAVELENGTH ABELIAN BACKGROUND FIELDS}

We have argued that for phenomenological applications the time-dependent instability field in Eq. (17) should simply be subtracted, leaving a simple expression for photon production in a nonequilibrium plasma. It is nevertheless interesting to explore the effect of the long-wavelength instability field, both from a theoretical point of view, as well as as a first step towards including background, classical fields to photon production through bremsstrahlung.

We will now calculate how the long-wavelength background fields modify photon emission, focusing on the case of an Abelian plasma. In particular we consider how the background fields modify medium-induced bremsstrahlung as seen in Fig. 1(c) which suffers from spurious divergences when one assumes a static, nonequilibrium plasma. Our setup is fairly general: The medium can be described by the $r r$ correlator in Eq. (17) but also by any other $r r$ correlator which has two different scales, fluctuating time-independent excitations with energy $g \Lambda$ and a time-dependent background field with energy $\xi g \Lambda, \xi \ll g$. This calculation also extends easily to jet-medium interaction and quasiparticle splitting as seen in Fig. 1(d). Our goal is to sum up nonperturbative effects of the background field at a given order in $l \Delta t \ll 1$ where $l \sim \xi g \Lambda$ is the small momentum of the background field and $\Delta t \sim 1 / g^{2} \Lambda$ is the time photon emission takes.

The two energy scales, i.e., the fluctuating field at $g \Lambda$ and the background field at $\xi g \Lambda$, affect photon emission in very different ways. The time for collinear bremsstrahlung of photons is $\approx 1 / g^{2} \Lambda$ which is very long compared to the time $1 / g \Lambda$ for a typical medium kick. Thus the medium kicks are ordered in time and diagrams with crossed rungs like in Fig. 3 are suppressed. On the other hand the long-wavelength background field has wavelength $\approx 1 / \xi g \Lambda$ which is much longer than the time for photon emission. Thus we must evaluate diagrams like in Fig. 3 for the background field. These diagrams are complicated because of the color factors and can only realistically be summed up in the case of an Abelian background field or a non-Abelian background field in the large $N_{c}$ limit where only planar diagrams contribute. We focus on the Abelian case here. Our goal is to do a calculation that includes both medium kicks and the background field as can be seen in Fig. 6 .

We make a few assumptions about the scales of the problem. First, we assume that the momentum $l$ of the longwavelength background field satisfies

$$
l \Delta t \ll 1
$$

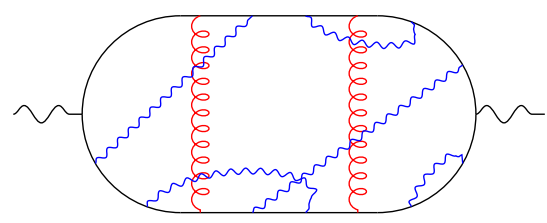

FIG. 6. A diagram for medium-induced photon production in the presence of a background field. The red gluons denote medium kicks at energy $g \Lambda$ which are time ordered. The blue lines denote kicks from the background field at energy $\xi g \Lambda$. They are not time ordered. 


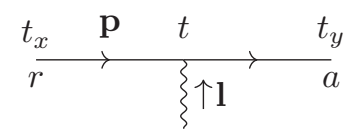

FIG. 7. A retarded propagator with one background field insertion.

where $\Delta t$ is the time the emission of a photon takes. In our case $\Delta t \sim 1 / g^{2} \Lambda$ so for instability fields $\xi \ll g$. Furthermore, we assume that $\gamma \Delta t \ll 1$ where $1 / \gamma$ is the time over which the background fields change appreciably. We also assume

$$
1 \ll \Lambda \Delta t
$$

where $\Lambda$ is the hard scale of the medium and $\Delta t$ is the time an emission takes. Medium-induced emission of photons or gluons takes time $\approx 1 / g^{2} \Lambda$ which is long enough to fulfill the condition. In general Eq. (47) is satisfied for off-shell photon emission with virtuality $Q^{2} \ll \Lambda^{2}$.

We finally assume that the wavelength of the background fields cannot be so long that it correlates two subsequent gluon emissions. In other words

$$
\frac{1}{\Lambda(\Delta t)^{2}} \ll l
$$

where $1 / \Lambda(\Delta t)^{2} \sim \Lambda / g^{4}$, the mean free path for gluon emission.

Quark propagators are modified in the presence of background fields. The bare retarded propagator is

$$
S_{\mathrm{ret}}^{(0)}\left(t_{x}, t_{y} ; \mathbf{p}\right)=\frac{1}{2} \theta(\Delta t)\left[e^{-i p \Delta t} \widehat{\boldsymbol{P}}-e^{i p \Delta t} \widetilde{\boldsymbol{P}}\right]
$$

where $\Delta t=t_{x}-t_{y}$ and $\widehat{P}=(1, \widehat{\mathbf{p}})$ and $\widetilde{P}=(-1, \widehat{\mathbf{p}})$ denote different polarizations. Adding one background field insertion, Fig. 7, gives

$$
S_{\mathrm{ret}}^{(1)}\left(t_{x}, t_{y} ; \mathbf{p} ; \mathbf{l}\right)=i g A_{\mu} \int d t S_{\mathrm{ret}}^{(0)}\left(t_{x}, t ; \mathbf{p}\right) \gamma^{\mu} S_{\mathrm{ret}}^{(0)}\left(t, t_{y} ; \mathbf{p}+\mathbf{l}\right) .
$$

We can take the background field $A^{\mu}$ out of the time integral since it changes slowly. This gives

$$
S_{\text {ret }}^{(1)}\left(t_{x}, t_{y} ; \mathbf{p} ; \mathbf{l}\right) \approx \frac{1}{2} \theta(\Delta t) e^{i p \Delta t}\left[\left(i g A_{\mu} \widehat{P}^{\mu}\right) \widehat{\boldsymbol{P}}\left(\Delta t-\frac{1}{2} i l_{\|}(\Delta t)^{2}\right)+\left(i g A_{\mu} \widetilde{P}^{\mu}\right) \widetilde{\boldsymbol{P}}\left(\Delta t+\frac{1}{2} i l_{\|}(\Delta t)^{2}\right)\right] .
$$

Here we have expanded in $\Delta t l_{\|} \ll 1$ with $l_{\|}=\widehat{\mathbf{p}} \cdot \mathbf{l}$. Terms with $\Delta t$ in Eq. (51) denote a potential phase rotation in the background field. The subleading term with $l_{\|}(\Delta t)^{2}$ gives the first derivative of the background field $A^{\mu}$ and thus denotes the effect of electromagnetic fields on photon emission. Higher-order terms are not amenable to evaluation using our methods. ${ }^{11}$

The retarded quark propagator with an arbitrary number of background field insertions is

$$
S_{\text {ret }}^{(n)}\left(t_{x}, t_{y} ; \mathbf{p} ;\left\{\mathbf{l}_{1}, \ldots, \mathbf{l}_{n}\right\}\right) \approx \frac{1}{2} \theta(\Delta t)\left[\left(i g A_{\mu} \widehat{P}^{\mu}\right)^{n} e^{-i p \Delta t} \widehat{\boldsymbol{P}} \prod_{i=1}^{n} \frac{e^{-i l_{i}^{\|} \Delta t}-1}{-i l_{i}^{\|}}+(-1)^{n+1}\left(i g A_{\mu} \widetilde{P}^{\mu}\right)^{n} e^{i p \Delta t} \widetilde{\boldsymbol{P}} \prod_{i=1}^{n} \frac{e^{i l_{i}^{\|} \Delta t}-1}{i l_{i}^{\|}}\right] .
$$

This simple form is achieved by summing over all the different permutations of attaching $n$ background field insertions. See Fig. 8 for an example with two background field insertions. The analogous expression for the advanced propagator has an overall minus sign and $\theta(-\Delta t)$ instead of $\theta(\Delta t)$.

Equation (52) can be derived by noting that the dependence on background field momentum is

$$
\int \frac{d \omega}{2 \pi} e^{-i \omega \Delta t} \frac{i}{\omega-p+i \epsilon} \frac{i}{\omega-\left|\mathbf{p}+\mathbf{l}_{1}\right|+i \epsilon} \cdots \frac{i}{\omega-\left|\mathbf{p}+\mathbf{l}_{1}+\cdots+\mathbf{l}_{n}\right|+i \epsilon}
$$

for $n$ ordered instability insertions. Performing the integral and expanding in $l_{i \|}=\hat{\mathbf{p}} \cdot \mathbf{l}_{i}$ gives a complicated expression. It is hugely simplified by summing over all permutations of attaching $n$ background field insertions, and using that

$$
\sum_{\text {permute }\left\{l_{1}, \ldots, l_{j}\right\}} \frac{1}{\left(l_{1}+\cdots+l_{j}\right)\left(l_{2}+\cdots+l_{j}\right) \ldots l_{j}}=\frac{1}{l_{1} \ldots l_{j}} .
$$

Finally, we must evaluate how the $r r$ propagator is modified in the presence of a long-wavelength background field. The bare $r r$ propagator in the time domain is

$$
\begin{aligned}
& S_{r r}^{(0)}\left(t_{x}, t_{y} ; \mathbf{k}\right)=\left[\frac{1}{2}-F_{q}(\mathbf{k})\right]\left[S_{\text {ret }}^{(0)}\left(t_{x}, t_{y} ; \mathbf{k}\right)-S_{\text {adv }}^{(0)}\left(t_{x}, t_{y} ; \mathbf{k}\right)\right]
\end{aligned}
$$

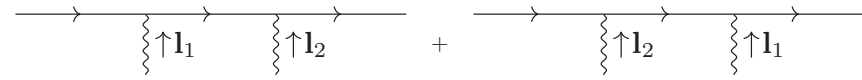

FIG. 8. A retarded propagator with two background field insertions. The different ways of attaching the insertions are summed over.

\footnotetext{
${ }^{11}$ The omitted terms in Eqs. (51) and (52) are in fact subleading. Cross terms like $\widehat{\boldsymbol{P}} \gamma^{\mu} \widetilde{\boldsymbol{P}}$ which denote spin flip in the background field give $\frac{1}{p} \ll l(\Delta t)^{2}$ after doing the time integral and can thus be ignored. Furthermore, we can ignore spin precession in the background fields. It will give spinor factors with $\widehat{P+L}^{\mu} \approx P^{\mu}+\mathcal{O}(l / \Lambda)$. After doing the time integral the spin precession correction gives a term $\mathcal{O}(l \Delta t / \Lambda)$ which is subleading to the terms in Eq. (52).
} 


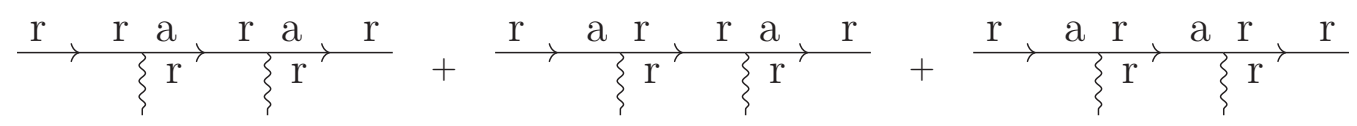

FIG. 9. The three different ways of ordering $r$ and $a$ indices on a $r r$ propagator with two background field insertions.

where

$$
F(\mathbf{k})= \begin{cases}f_{q}(\mathbf{k}), & \text { for } \widehat{K} \\ 1-f_{\bar{q}}(-\mathbf{k}), & \text { for } \widetilde{K}\end{cases}
$$

describes the momentum distribution for incoming quarks and outgoing antiquarks, respectively. There are many ways to add background field insertions in the $r a$ basis. As an example Fig. 9 shows the three possible ways of including two background field insertions. To find them we have used that a background field insertion has index $r$, that each vertex has an odd number of $a$ indices, and that bare $a a$ propagators vanish [14]. Assuming that the momentum distributions in each propagator are the same as our order of approximation, $f_{q}(\mathbf{k}) \approx f_{q}\left(\mathbf{k}+\mathbf{l}_{1}\right) \approx f_{q}\left(\mathbf{k}+\mathbf{l}_{1}+\mathbf{l}_{2}\right),{ }^{12}$ and using Eq. (55), most of the terms cancel [13]. We end up with

$$
S_{r r}^{(2)}=\left[\frac{1}{2}-F_{q}(\mathbf{k})\right]\left(S_{\text {ret }}^{(2)}-S_{\text {adv }}^{(2)}\right) .
$$

A similar cancellation takes place for any number of background field insertions so that in the end

$$
\begin{aligned}
& S_{r r}^{(n)}\left(t_{x}, t_{y} ; \mathbf{p} ;\left\{\mathbf{l}_{1}, \ldots, \mathbf{l}_{n}\right\}\right) \\
&=\left[\frac{1}{2}-F(\mathbf{k})\right][ S_{\text {ret }}^{(n)}\left(t_{x}, t_{y} ; \mathbf{p} ;\left\{\mathbf{l}_{1}, \ldots, \mathbf{l}_{n}\right\}\right) \\
&\left.-S_{\text {adv }}^{(n)}\left(t_{x}, t_{y} ; \mathbf{p} ;\left\{\mathbf{l}_{1}, \ldots, \mathbf{l}_{n}\right\}\right)\right] .
\end{aligned}
$$

\section{MEDIUM-INDUCED PHOTON EMISSION IN ABELIAN BACKGROUND FIELDS}

We turn to evaluating photon emission in an Abelian background field. For simplicity, we begin by only considering the long-wavelength background field with momentum $\xi g \Lambda$, considering medium kicks with momentum $g \Lambda$ below.

\footnotetext{
${ }^{12}$ By making the approximation $f_{q}(\mathbf{k}+\mathbf{l}) \approx f_{q}(\mathbf{k})$ we ignore how quarks are rotated in the background field during emission. This correction is of order $\mathbf{I} \cdot \nabla f(\mathbf{p}) \sim \frac{l}{\Lambda} f(\mathbf{p})$. Such terms have a combination of retarded and advanced propagator with no simple time ordering. The time integral at the vertex with momentum contribution 1 will thus give $T$, the time that has passed since the initial conditions that specified the momentum distribution $f(\mathbf{p})$. Choosing $T \gtrsim \Delta t$ so that the momentum distribution describes the quarks just before they emit the photon, it is easy to see that the correction is subleading to Eq. (52).
}

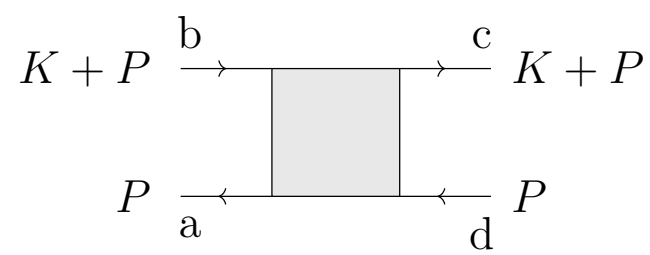

FIG. 10. Definition of the four point quark function $S_{a b c d}$.
On-shell photon emission from on-shell quarks is kinematically suppressed in the absence of kicks from a background field or a medium. The rate of on-shell photon emission is given by $\Pi_{12}^{\gamma}$ which goes like the four-point quark correlator $S_{1122}$; see Fig. 10. We show in Appendix B that

$$
S_{1122}=2 F(P+K)[1-F(P)] \operatorname{Re} S_{\text {rraa }}
$$

where we have gone to the $r a$ basis in the closed-time path formalism [32] defined by

$$
\psi_{r}=\frac{\psi_{1}+\psi_{2}}{2}, \quad \psi_{a}=\psi_{1}-\psi_{2} .
$$

The momentum factors in Eq. (59) describe different channels. As an example with $p^{0}>0$ we get $f_{q}(\mathbf{p}+\mathbf{k})\left[1-f_{q}(\mathbf{p})\right]$ which denotes a quark with momentum $\mathbf{p}+\mathbf{k}$ emitting a photon with momentum $\mathbf{k}$ through bremsstrahlung. The rate of emitting an on-shell photon with momentum $k$ through quark bremsstrahlung then goes like

$$
\begin{aligned}
i \Pi_{12 \mu}^{\mu}(\mathbf{k})= & \frac{e^{2}}{4} \operatorname{Tr}\left[\gamma_{\mu} \widehat{K} \gamma^{\mu} \widehat{K}\right] 2 f_{q}(\mathbf{p}+\mathbf{k})\left[1-f_{q}(\mathbf{p})\right] \\
& \times \operatorname{Re} \int d\left(t_{x}-t_{y}\right) e^{i k\left(t_{x}-t_{y}\right)} \theta\left(t_{x}-t_{y}\right) \\
& \times e^{-i(|\mathbf{p}+\mathbf{k}|-p)\left(t_{x}-t_{y}\right)}
\end{aligned}
$$

as can be seen in Fig. 11. The frequency integral gives $\delta(k-|\mathbf{p}+\mathbf{k}|+p)$ which vanishes under integration over $\mathbf{p}$ for an on-shell photon. This is simply because the emission is kinematically suppressed.

We now turn on the background field and see whether on-shell photon emission becomes possible. Since we have assumed that $\langle A\rangle=0$ but $G_{r r}=\frac{1}{2}\langle\{A, A\}\rangle \neq 0$ we must pair up the background field insertions into $r r$ two-point functions to account for fluctuations in the background field. An example of a contribution can be seen in Fig. 3.

The upper quark rail with momentum $\mathbf{p}+\mathbf{k}$ becomes

$$
\begin{aligned}
& S_{\mathrm{ret}}^{n_{1}}\left(t_{x}, t_{y} ; \mathbf{p}+\mathbf{k} ;\left\{\mathbf{l}_{1}, \ldots\right\}\right) \\
& \quad=S_{\mathrm{ret}}^{(0)}\left(t_{x}, t_{y} ; \mathbf{p}+\mathbf{k}\right)\left(i g A_{\mu} \widehat{K}^{\mu}\right)^{n_{1}} \prod_{j=1}^{n_{1}} \frac{e^{-\widehat{\widehat{\mathbf{k}}} \cdot \mathbf{l}_{j}\left(t_{x}-t_{y}\right)}-1}{-i \widehat{\mathbf{k}} \cdot \mathbf{l}_{j}}
\end{aligned}
$$

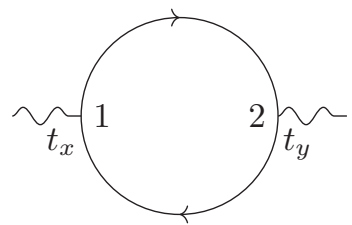

FIG. 11. Photon self-energy diagram, without a background field or a medium. 
after summing over all possible permutations of $n_{1}$ background field insertions. Similarly, the lower quark rail with momentum $\mathbf{k}$ becomes

$$
\begin{aligned}
S_{\mathrm{adv}}^{n_{2}}\left(t_{y}, t_{x} ; \mathbf{p}+\mathbf{k}+\sum \mathbf{l}_{i} ;\left\{\tilde{\mathbf{l}}_{1}, \ldots\right\}\right) \\
=S_{\mathrm{ret}}^{(0)}\left(t_{y}, t_{x} ; \mathbf{p}+\mathbf{k}\right) e^{-i \sum_{i} \widehat{\mathbf{k}} \cdot \mathbf{l}_{i}\left(t_{y}-t_{x}\right)} \\
\quad \times\left(i g A_{\mu} \widehat{K}^{\mu}\right)^{n_{2}} \prod_{j=1}^{n_{2}} \frac{e^{-\hat{\mathbf{k}} \cdot \tilde{j}_{j}\left(t_{y}-t_{x}\right)}-1}{-\hat{i \mathbf{k}} \cdot \tilde{\mathbf{l}}_{j}},
\end{aligned}
$$

with $n_{2}$ instability insertions. The extra factor of $e^{-i \sum_{i} \widehat{\mathbf{k}} \cdot \mathbf{l}_{i}\left(t_{y}-t_{x}\right)}$ arises because the momentum flow into the advanced propagator is $\mathbf{p}+\mathbf{k}+\sum \mathbf{l}_{i}$ where $\mathbf{l}_{i}$ comes from the retarded propagator.

When pairing up background insertions we must integrate over the momenta in $r r$ propagators, $\mathbf{l}$ and $\widetilde{\mathbf{l}}$. Pairing up two background field insertions on the upper quark rail gives a factor

$$
\begin{aligned}
D:= & -g^{2} \widehat{K}_{\mu} \widehat{K}_{v} \int \frac{d^{3} l}{(2 \pi)^{3}} G_{r r}^{\mu v}(T ; \mathbf{l}) \\
& \times \frac{e^{-i \widehat{\mathbf{k}} \cdot \mathbf{l}\left(t_{x}-t_{y}\right)}-1}{-\hat{i} \widehat{\mathbf{k}} \cdot \mathbf{l}} \frac{e^{i \mathbf{k} \cdot \mathbf{l}\left(t_{x}-t_{y}\right)}-1}{i \widehat{\mathbf{k}} \cdot \mathbf{l}} \\
\approx & -g^{2} \widehat{K}_{\mu} \widehat{K}_{v} \int \frac{d^{3} l}{(2 \pi)^{3}} G_{r r}^{\mu \nu}(T ; \mathbf{l})\left[(\Delta t)^{2}-\frac{1}{12} l^{2}(\Delta t)^{4}\right]
\end{aligned}
$$

where we used that the momentum flow is $\mathbf{l}$ in one insertion, and $-\mathbf{l}$ in the other insertion. The first term, $(\Delta t)^{2}$, describes a phase shift and the second term describes how the dispersion relation changes because of fluctuating background fields. Pairing up two background field insertions on the lower quark rail gives the same factor $D$. Finally, pairing up an insertion from the upper rail and an insertion from the lower rail gives

$$
\begin{aligned}
& -g^{2} \widehat{K}_{\mu} \widehat{K}_{v} \int \frac{d^{3} l}{(2 \pi)^{3}} G_{r r}^{\mu v}(T ; \mathbf{l}) \frac{e^{-i \widehat{\mathbf{k}} \cdot \mathbf{l}\left(t_{x}-t_{y}\right)}-1}{-\hat{\mathbf{k}} \cdot \mathbf{l}} \\
& \quad \times e^{-i \widehat{\mathbf{k}} \cdot \mathbf{l}\left(t_{y}-t_{x}\right)} \frac{e^{\widehat{i \mathbf{k}} \cdot \mathbf{l}\left(t_{y}-t_{x}\right)}-1}{i \widehat{\mathbf{k}} \cdot \mathbf{l}}
\end{aligned}
$$

which has the value $-D$.

We must now sum over all possible ways of attaching background field insertions to the two quark rails. A typical diagram can be seen in Fig. 3. Fortunately, we have already summed over all ways of ordering field insertions on each quark rail in Eqs. (62) and (63). Thus we only need to sum over the number of insertions on each rail and the different ways of joining them in $r r$ propagators. Assuming that there are $m_{1} r r$ propagators where both ends are on the upper quark rail, $m_{2}$ propagators with both ends on the lower quark rail, and $m_{3}$ pairs that join the two propagator, the time dependence becomes

$$
\begin{aligned}
& \theta\left(t_{x}-t_{y}\right) e^{-i(|\mathbf{p}+\mathbf{k}|-p)\left(t_{x}-t_{y}\right)} \\
& \quad \times \sum_{n=0}^{\infty} \sum_{\substack{m_{1}, m_{2}, m_{3} \geqslant 0 \\
m_{1}+m_{2}+m_{3}=n}} \frac{1}{m_{1} ! m_{2} ! m_{3} !} \frac{1}{2^{m_{1}} 2^{m_{2}}} D^{m_{1}} D^{m_{2}}(-D)^{m_{3}} .
\end{aligned}
$$

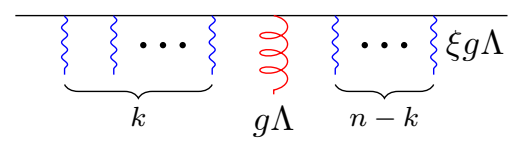

FIG. 12. A retarded propagator with one HTL medium kick and $n$ background field insertions.

There is a total of $2 n$ background field insertions. The combinatorial factors account for the fact that the diagram remains the same after interchanging different propagators between the same rails or interchanging the ends of a propagator. (We do not divide by $2^{m_{3}}$ since we have not permuted instability insertions between the rails.) The combinatorial sum in Eq. (66) gives

$$
\sum_{n=0}^{\infty} \frac{1}{2^{n} n !}(D+D-2 D)^{n}=1 .
$$

Thus the total contribution of the background field cancels out in the Abelian case. The same cancellation takes place in the other channels, namely, for an antiquark emitting a photon and in quark-antiquark pair annihilation.

The effect of a long-wavelength background field on photon emission is vanishing to our order of approximation. This is true in the absence of a medium but it turns out to be equally true when there is a medium kicking the quarks back and forth in the transverse plane. To show this, we need retarded propagators including both medium kicks and the effect of a long-wavelength background field. With a medium kick at time $t$ and $k$ background field insertions before the kick and $n-k$ insertions after the kick (see Fig. 12), the quark propagator becomes

$$
\begin{aligned}
S_{\mathrm{ret}}^{(k)}( & \left.t_{x}, t ; \mathbf{p} ;\left\{\mathbf{l}_{1}, \ldots, \mathbf{l}_{k}\right\}\right) \mathcal{I}(t ; \mathbf{q}) \\
& \times S_{\mathrm{ret}}^{(n-k)}\left(t, t_{y} ; \mathbf{p}+\mathbf{q}+\mathbf{l}_{1}+\cdots+\mathbf{l}_{k} ;\left\{\mathbf{l}_{k+1}, \ldots, \mathbf{l}_{n}\right\}\right) \\
= & S_{\mathrm{ret}}^{(0)}\left(t_{x}, t ; \mathbf{p}\right) \mathcal{I}(t ; \mathbf{q}) S_{\mathrm{ret}}^{(0)}\left(t, t_{y} ; \mathbf{p}+\mathbf{q}\right) \\
& \times\left(i g A_{\mu} \widehat{P}^{\mu}\right)^{n} \prod_{i=1}^{k} X\left(\mathbf{l}_{i}\right) \prod_{j=k+1}^{n} Y\left(\mathbf{l}_{i}\right)
\end{aligned}
$$

where $\mathcal{I}$ is the vertex factor for the medium kick and $\mathbf{q}$ is the momentum flow in the kick. We have defined

$$
X\left(\mathbf{l}_{i}\right)=\frac{e^{-i \widehat{\mathbf{p}} \cdot \mathbf{l}_{i}\left(t_{x}-t\right)}-1}{-i \widehat{\mathbf{p}} \cdot \mathbf{l}_{i}} e^{-i \widehat{\mathbf{p}} \cdot \mathbf{l}_{i}\left(t-t_{y}\right)}
$$

for background field insertions left of the kick and

$$
Y\left(\mathbf{l}_{j}\right)=\frac{e^{-i \widehat{\mathbf{p}} \cdot \mathbf{l}_{j}\left(t-t_{y}\right)}-1}{-i \widehat{\mathbf{p}} \cdot \mathbf{l}_{j}}
$$

for insertions right of the kick. We have furthermore used that $\widehat{\mathbf{p}+\mathbf{q}} \approx \widehat{\mathbf{p}}$ to avoid corrections of order $g l(\Delta t)^{2}$ to Eq. (68).

We now sum over all ways of attaching the $n$ background field insertions, either before or after the medium kick. This gives

$$
\begin{aligned}
& S_{\text {ret }}^{(0)}\left(t_{x}, t ; \mathbf{p}\right) \mathcal{I}(t) S_{\text {ret }}^{(0)}\left(t, t_{y} ; \mathbf{p}+\mathbf{q}\right) \\
& \quad \times\left(i g A_{\mu} \widehat{P}^{\mu}\right)^{n} \prod_{i=1}^{n}\left[X\left(\mathbf{l}_{i}\right)+Y\left(\mathbf{l}_{j}\right)\right]
\end{aligned}
$$




$$
\begin{aligned}
= & S_{\text {ret }}^{(0)}\left(t_{x}, t ; \mathbf{p}\right) \mathcal{I}(t) S_{\text {ret }}^{(0)}\left(t, t_{y} ; \mathbf{p}+\mathbf{q}\right) \\
& \times\left(i g A_{\mu} \widehat{P}^{\mu}\right)^{n} \prod_{i=1}^{n} \frac{e^{-i \widehat{\mathbf{p}} \mathbf{l}_{i}\left(t_{x}-t_{y}\right)}-1}{-i \widehat{\mathbf{p}} \cdot \mathbf{l}_{i}} .
\end{aligned}
$$

The effect of the instabilities is a factor which does not depend on the time of the medium kick. This argument can clearly be extended for any number of medium kicks. Thus, the effect of medium kicks and $n$ background field insertions factorizes and the dependence on the background field strength is exactly the same as in the case without a medium. The same argument that lead to Eq. (67) then shows that the background field does not affect photon emission at leading and next-to-leading order in $l \Delta t$ and the rate is given by Eq. (23), independent of the occupation density of the instabilities that was discussed in Sec. III.

\section{CONCLUSIONS}

Nonequilibrium QCD plasmas at weak coupling contain instabilities which lead to exponential growth in soft gluon density with time. This makes the plasma inherently time dependent. Therefore, quantum field theory calculations that assume a static nonequilibrium plasma do not work. In particular, assuming a static plasma leads to spurious divergences in the rate of medium-induced jet splitting or photon production in the plasma.

In this paper, we consider a nonequilibrium setup with a small anisotropy, $\xi \ll g$, in the initial momentum distribution of quasiparticles. Using tools of nonequilibrium quantum field theory, we derive the time dependence of the retarded correlator, Eq. (14), and $r r$ correlator, Eq. (17), at early times. As expected, the $r r$ correlator shows exponential growth in the soft gluon density because of instabilities. We furthermore derive a collision kernel, Eq. (20), in our setup. This kernel is the probability for a photon-emitting quark to get a transverse momentum kick from a gluon in the medium. We show that the collision kernel factorizes into kicks from a fluctuating cloud of soft gluons, and a time-dependent instability contribution with exponential growth. Crucially, the instability contribution is finite, meaning that spurious divergences in the rate of photon production are cured when time dependence of the plasma is taken into account. At higher values of anisotropy the collision kernel changes rapidly while a photon is being emitted and some of our approximations break down. But importantly, we show that the spurious divergences are also cured for higher anisotropy when time dependence is included.

The time-dependent instability modes in the collision kernel have a complicated effect on photon emission. Due to their long wavelength the transverse momentum kicks of the instability modes are not ordered in time, making their inclusion more challenging. We take a step in that direction by showing that in an Abelian plasma the instabilities' effect on photon production vanishes up to next-to-leading order in $l \Delta t \ll 1$ where $l$ is the energy scale of the instabilities and $\Delta t$ is the time needed to emit a photon.

For phenomenological purposes we suggest a simple prescription in which the instability contribution is omitted when evaluating the rate of photon production and jet splitting. Our argument is twofold. First, we have shown that there are no spurious divergences when time dependence is taken into account, meaning that instabilities are not enhanced through divergences. Second, the remaining effect of instability modes comes through the $r r$ correlator which measures their occupation density. Thus the instability modes can be omitted when their occupation is sufficiently low. Indeed, recent numerical work using classical-statistical field theory suggests that instability modes are no longer highly occupied once the hydrodynamical stage is reached [28,29], justifying our prescription.

Using our phenomenological prescription one gets rates that only depend on the instantaneous properties of the medium; see Eq. (23). They contain both nonequilibrium momentum distributions and the nonequilibrium, fluctuating soft gluon cloud which has not yet been evaluated. The rate equations can then be solved numerically whenever a momentum distribution of quarks and gluons is specified; see [30,31]. Thus it becomes possible to calculate the effect of shear viscous flow on jet evolution in the plasma, as well as photon production, through all leading-order channels. Combined with a hydrodynamical model of the QGP fluid, this supports the possibility of using jet physics and photons to constrain the magnitude of the viscosity of QGP.

\section{ACKNOWLEDGMENTS}

This work was supported in part by the Natural Sciences and Engineering Research Council of Canada. S.H. gratefully acknowledges a scholarship from the Fonds de Recherche du Québec-Nature et Technologies.

\section{APPENDIX A: EVALUATION OF CORRELATORS}

We begin by deriving the retarded propagator in Eq. (14) in full detail. We start from Eq. (29) and evaluate it term by term by inserting principal values as in Eq. (31). The first term becomes

$$
\begin{aligned}
& -\int \frac{d k_{1}}{2 \pi} \int \frac{d k_{2}}{2 \pi} \int_{\alpha} \frac{d k_{3}}{2 \pi} G_{\mathrm{ret}}^{0}\left(k_{1}\right) \Pi_{\mathrm{ret}}\left(k_{2}\right) G_{\mathrm{ret}}\left(k_{3}\right) e^{-i k_{1}(x-y)} \\
& \quad \times \frac{1}{8}\left[\left(\frac{i}{k_{1}-k_{2}+i \epsilon_{1}-i \epsilon_{2}}+\frac{i}{k_{1}-k_{2}+i \epsilon_{1}+i \epsilon_{2}}\right)\left(\frac{i}{k_{1}-k_{3}+i \epsilon_{1}-i \epsilon_{3}}+\frac{i}{k_{1}-k_{3}+i \epsilon_{1}+i \epsilon_{3}}\right)\right. \\
& \left.\quad+\left(\frac{i}{k_{1}-k_{2}-i \epsilon_{1}-i \epsilon_{2}}+\frac{i}{k_{1}-k_{2}-i \epsilon_{1}+i \epsilon_{2}}\right)\left(\frac{i}{k_{1}-k_{3}-i \epsilon_{1}-i \epsilon_{3}}+\frac{i}{k_{1}-k_{3}-i \epsilon_{1}+i \epsilon_{3}}\right)\right] .
\end{aligned}
$$


We can continue the $k_{3}$ integral by adding a semicircle in the upper half plane with a large radius; see Fig. 2(b). Using the residue theorem we avoid all poles of $G_{\text {ret }}$ so only the poles in the square bracket contribute. We similarly continue the $k_{2}$ to the upper half plane. This gives

$$
\int_{\mathbb{R}} \frac{d k_{1}}{2 \pi} G_{\text {ret }}^{0}\left(k_{1}\right) \Pi_{\text {ret }}\left(k_{1}\right) G_{\text {ret }}\left(k_{1}\right) e^{-i k_{1}(x-y)} \frac{1}{8}\left\{\theta\left(\epsilon_{2}-\epsilon_{1}\right) \theta\left(\epsilon_{3}-\epsilon_{1}\right)+\left[1+\theta\left(\epsilon_{1}-\epsilon_{2}\right)\right]\left[1+\theta\left(\epsilon_{1}-\epsilon_{3}\right)\right]\right\},
$$

where the integration contour is simply the real line. In a similar fashion, the second term in Eq. (29) becomes

$$
\int_{\mathbb{R}} \frac{d k}{2 \pi} G_{\text {ret }}^{0}(k) \Pi_{\text {ret }}(k) G_{\text {ret }}(k) e^{-i k(x-y)} \frac{1}{8}\left\{\theta\left(\epsilon_{1}-\epsilon_{2}\right) \theta\left(\epsilon_{3}-\epsilon_{2}\right)+\left[1+\theta\left(\epsilon_{2}-\epsilon_{1}\right)\right]\left[1+\theta\left(\epsilon_{2}-\epsilon_{3}\right)\right]\right\} .
$$

The third term in Eq. (29) is slightly trickier to evaluate. We write the $k_{3}$ integration contour as $\int_{\alpha}=\int_{\mathbb{R}}+\sum_{i} \int_{\gamma_{i}}$ where $\gamma_{i}$ go around the instability poles in the upper half complex plane; see Fig. 2(c). The part with the real line integration gives

$$
\int_{\mathbb{R}} \frac{d k_{3}}{2 \pi} G_{\mathrm{ret}}^{0}\left(k_{3}\right) \Pi_{\mathrm{ret}}\left(k_{3}\right) G_{\mathrm{ret}}\left(k_{3}\right) e^{-i k_{3}(x-y)} \frac{1}{8}\left\{\left[1+\theta\left(\epsilon_{3}-\epsilon_{1}\right)\right]\left[1+\theta\left(\epsilon_{3}-\epsilon_{2}\right)\right]+\theta\left(\epsilon_{1}-\epsilon_{3}\right) \theta\left(\epsilon_{2}-\epsilon_{3}\right)\right\}
$$

after doing the $k_{1}$ and $k_{2}$ integrals. The part with integration over the $\gamma_{i}$ contour can be done explicitly, giving

$$
\sum_{i} \int_{\gamma_{i}} \frac{d k}{2 \pi} G_{\mathrm{ret}}^{0}(k) \Pi_{\mathrm{ret}}(k) G_{\mathrm{ret}}(k) e^{-i k(x-y)}
$$

We are finally in a position to find the retarded propagator. Adding up the contributions of Eqs. (A2), (A3), (A4), and (A5) and using identities of $\theta$ functions we get

$$
\int_{\alpha} \frac{d k}{2 \pi} G_{\text {ret }}^{0}(k) \Pi_{\text {ret }}(k) G_{\text {ret }}(k) e^{-i k(x-y)}
$$

which leads directly to Eq. (32) and thus to Eq. (14) as we wanted to show.

We next evaluate the $r r$ correlator. Specifically, we will show how Eq. (39) follows from Eq. (36) using the approximations described in Sec. IV. Just like for the evaluation of $G_{\text {ret }}$ there are no poles when $k_{1}=k_{2}$ or $k_{2}=k_{3}$ which allows us to insert principal values. This gives

$$
\begin{aligned}
G_{r r}(x, y)= & \int_{\alpha} \frac{d k_{1}}{2 \pi} \int \frac{d k_{2}}{2 \pi} \int_{\widetilde{\alpha}} \frac{d k_{3}}{2 \pi}\left[e^{-i k_{2}(x-y)}-e^{-i k_{1} x} e^{i k_{2} y}-e^{-i k_{2} x} e^{i k_{3} y}+e^{-i k_{1} x} e^{i k_{3} y}\right] \\
& \times \frac{1}{8}\left[\left(\frac{1}{k_{2}-k_{1}+i \epsilon_{2}-i \epsilon_{1}}+\frac{1}{k_{2}-k_{1}+i \epsilon_{2}+i \epsilon_{1}}\right)\left(\frac{1}{k_{2}-k_{3}+i \epsilon_{2}-i \epsilon_{3}}+\frac{1}{k_{2}-k_{3}+i \epsilon_{2}+i \epsilon_{3}}\right)\right. \\
& \left.+\left(\frac{1}{k_{2}-k_{1}-i \epsilon_{2}-i \epsilon_{1}}+\frac{1}{k_{2}-k_{1}-i \epsilon_{2}+i \epsilon_{1}}\right)\left(\frac{1}{k_{2}-k_{3}-i \epsilon_{2}-i \epsilon_{3}}+\frac{1}{k_{2}-k_{3}-i \epsilon_{2}+i \epsilon_{3}}\right)\right] \\
& \times\left(\widehat{G}_{\mathrm{ret}}\left(k_{1}\right)+\sum_{i} \frac{A_{i}}{k_{1}-a_{i}}\right) \Pi_{a a}\left(k_{2}\right)\left(\widehat{G}_{\mathrm{adv}}\left(k_{3}\right)+\sum_{j} \frac{A_{j}^{*}}{k_{3}-a_{j}^{*}}\right),
\end{aligned}
$$

where we have substituted the scale separation of Eqs. (37) and (38).

The evaluation of Eq. (A7) depends on the scale one is working at. We begin by evaluating terms at the scale $g \Lambda$, i.e., terms with $\widehat{G}_{\text {ret }}$ and $\widehat{G}_{\text {adv }}$. We do this one exponential at a time. The first exponential term (i.e., all terms with $e^{-i k_{2}(x-y)}$ ) can be evaluated exactly by continuing the $k_{1}$ integral to the upper half complex plane, the $k_{3}$ to the lower half complex plane, and applying the residue theorem. Then all poles of $\widehat{G}_{\text {ret }}$ and $\widehat{G}_{\text {adv }}$ are avoided and one gets

$$
\int \frac{d k_{2}}{2 \pi} \widehat{G}_{\mathrm{ret}}\left(k_{2}\right) \Pi_{a a}\left(k_{2}\right) \widehat{G}_{\mathrm{adv}}\left(k_{3}\right) e^{-i k_{2}(x-y)} \frac{1}{8}\left\{\theta\left(\epsilon_{3}-\epsilon_{2}\right)\left[1+\theta\left(\epsilon_{2}-\epsilon_{1}\right)\right]+\theta\left(\epsilon_{1}-\epsilon_{2}\right)\left[1+\theta\left(\epsilon_{2}-\epsilon_{3}\right)\right]\right\} .
$$


In the second exponential term (i.e., all terms with $-e^{-i k_{1} x} e^{i k_{2} y}$ ) in Eq. (A7) we continue the $k_{3}$ integral to the lower half plane, giving

$$
\begin{aligned}
& -i \int_{\alpha} \frac{d k_{1}}{2 \pi} \int \frac{d k_{2}}{2 \pi} \widehat{G}_{\mathrm{ret}}\left(k_{1}\right) \Pi_{a a}\left(k_{2}\right) \widehat{G}_{\mathrm{adv}}\left(k_{2}\right) e^{-i k_{1} x} e^{i k_{2} y} \frac{1}{8}\left[\theta\left(\epsilon_{3}-\epsilon_{2}\right)\left(\frac{1}{k_{2}-k_{1}+i \epsilon_{2}-i \epsilon_{1}}+\frac{1}{k_{2}-k_{1}+i \epsilon_{2}+i \epsilon_{1}}\right)\right. \\
& \left.+\left[1+\theta\left(\epsilon_{2}-\epsilon_{3}\right)\right]\left(\frac{1}{k_{2}-k_{1}-i \epsilon_{2}-i \epsilon_{1}}+\frac{1}{k_{2}-k_{1}-i \epsilon_{2}+i \epsilon_{1}}\right)\right]
\end{aligned}
$$

exactly. In order to evaluate the $k_{1}$ integral we need to use our approximations. Because of the exponential we must continue the contour to the lower half complex plane. Applying the residue theorem we get a contribution from all poles and branch cuts of $\widehat{G}_{\text {ret }}$ but they all contain a factor $e^{-i b x}$ with $b \sim g \Lambda$ and can thus be dropped according to our approximations. Thus only poles with $k_{2}=k_{1}$ contribute, giving

$$
\int \frac{d k_{2}}{2 \pi} \widehat{G}_{\mathrm{ret}}\left(k_{2}\right) \Pi_{a a}\left(k_{2}\right) \widehat{G}_{\mathrm{adv}}\left(k_{2}\right) e^{-i k_{2}(x-y)} \frac{1}{8}\left\{\theta\left(\epsilon_{1}-\epsilon_{2}\right) \theta\left(\epsilon_{3}-\epsilon_{2}\right)+\left[1+\theta\left(\epsilon_{2}-\epsilon_{3}\right)\right]\left[1+\theta\left(\epsilon_{2}-\epsilon_{1}\right)\right]\right\}
$$

In the same way, the third exponential term in Eq. (A7) is

$$
\int \frac{d k_{2}}{2 \pi} \widehat{G}_{\mathrm{ret}}\left(k_{2}\right) \Pi_{a a}\left(k_{2}\right) \widehat{G}_{\mathrm{adv}}\left(k_{2}\right) e^{-i k_{2}(x-y)} \frac{1}{8}\left\{\theta\left(\epsilon_{1}-\epsilon_{2}\right) \theta\left(\epsilon_{3}-\epsilon_{2}\right)+\left[1+\theta\left(\epsilon_{2}-\epsilon_{1}\right)\right]\left[1+\theta\left(\epsilon_{2}-\epsilon_{3}\right)\right]\right\}
$$

and the fourth exponential is

$$
\int \frac{d k_{2}}{2 \pi} \widehat{G}_{\mathrm{ret}}\left(k_{2}\right) \Pi_{a a}\left(k_{2}\right) \widehat{G}_{\mathrm{adv}}\left(k_{2}\right) e^{-i k_{2}(x-y)} \frac{1}{8}\left\{\theta\left(\epsilon_{1}-\epsilon_{2}\right)\left[1+\theta\left(\epsilon_{2}-\epsilon_{3}\right)\right]+\theta\left(\epsilon_{3}-\epsilon_{2}\right)\left[1+\theta\left(\epsilon_{2}-\epsilon_{1}\right)\right]\right\}
$$

Adding up the different terms in Eqs. (A8), (A10), (A11), and (A12) and using identities for $\theta$ functions, we get that the contribution to the $r r$ propagator at the scale $g \Lambda$ is

$$
\approx \int \frac{d k}{2 \pi} \widehat{G}_{\mathrm{ret}}(k) \Pi_{a a}(k) \widehat{G}_{\mathrm{adv}}(k) e^{-i k(x-y)} .
$$

We next turn to evaluating terms in Eq. (A7) at the scale $\xi g \Lambda$, i.e., the contribution of instability poles in the retarded and advanced propagators. As before the contribution of the first exponential is

$$
\sum_{i, j} \int \frac{d k_{2}}{2 \pi} \frac{A_{i}}{k_{2}-a_{i}} \Pi_{a a}\left(k_{2}\right) \frac{A_{j}^{*}}{k_{2}-a_{j}^{*}} e^{-i k_{2}(x-y)} \frac{1}{8}\left\{\theta\left(\epsilon_{3}-\epsilon_{2}\right)\left[1+\theta\left(\epsilon_{2}-\epsilon_{1}\right)\right]+\theta\left(\epsilon_{1}-\epsilon_{2}\right)\left[1+\theta\left(\epsilon_{2}-\epsilon_{3}\right)\right]\right\}
$$

In the second exponential in Eq. (A7) we continue the $k_{3}$ integral to the lower half plane to get

$$
\begin{aligned}
- & i \sum_{i, j} \int_{\alpha} \frac{d k_{1}}{2 \pi} \int \frac{d k_{2}}{2 \pi} \frac{A_{i}}{k_{1}-a_{i}} \Pi_{a a}\left(k_{2}\right) \frac{A_{j}^{*}}{k_{2}-a_{j}^{*}} e^{-i k_{1} x} e^{i k_{2} y} \\
& \times \frac{1}{8}\left[\theta\left(\epsilon_{3}-\epsilon_{2}\right)\left(\frac{1}{k_{2}-k_{1}+i \epsilon_{2}-i \epsilon_{1}}+\frac{1}{k_{2}-k_{1}+i \epsilon_{2}+i \epsilon_{1}}\right)\right. \\
& \left.\times\left[1+\theta\left(\epsilon_{2}-\epsilon_{3}\right)\right]\left(\frac{1}{k_{2}-k_{1}-i \epsilon_{2}-i \epsilon_{1}}+\frac{1}{k_{2}-k_{1}-i \epsilon_{2}+i \epsilon_{1}}\right)\right] .
\end{aligned}
$$

Now when we continue the $k_{1}$ integral to the lower half plane we get a contribution from $k_{1}=k_{2}$ as well as a contribution from $k_{1}=a_{i}$ leading to

$$
\begin{aligned}
& \sum_{i, j} \int \frac{d k_{2}}{2 \pi} \frac{A_{i}}{k_{2}-a_{i}} \Pi_{a a}\left(k_{2}\right) \frac{A_{j}^{*}}{k_{2}-a_{j}^{*}} e^{-i k_{2}(x-y)} \\
& \quad \times \frac{1}{8}\left\{\theta\left(\epsilon_{1}-\epsilon_{2}\right) \theta\left(\epsilon_{3}-\epsilon_{2}\right)+\left[1+\theta\left(\epsilon_{2}-\epsilon_{3}\right)\right]\left[1+\theta\left(\epsilon_{2}-\epsilon_{1}\right)\right]\right\} \\
& \quad-\frac{1}{2} \sum_{i, j} \int \frac{d k_{2}}{2 \pi} \frac{A_{i}}{k_{2}-a_{i}} \Pi_{a a}\left(k_{2}\right) \frac{A_{j}^{*}}{k_{2}-a_{j}^{*}} e^{-i a_{i} x} e^{i k_{2} y} .
\end{aligned}
$$


Similarly, the third exponential in Eq. (A7) is

$$
\begin{aligned}
& \sum_{i, j} \int \frac{d k_{2}}{2 \pi} \frac{A_{i}}{k_{2}-a_{i}} \Pi_{a a}\left(k_{2}\right) \frac{A_{j}^{*}}{k_{2}-a_{j}^{*}} e^{-i k_{2}(x-y)} \\
& \quad \times \frac{1}{8}\left\{\theta\left(\epsilon_{1}-\epsilon_{2}\right) \theta\left(\epsilon_{3}-\epsilon_{2}\right)+\left[1+\theta\left(\epsilon_{2}-\epsilon_{1}\right)\right]\left[1+\theta\left(\epsilon_{2}-\epsilon_{3}\right)\right]\right\} \\
& \quad-\frac{1}{2} \sum_{i, j} \int \frac{d k_{2}}{2 \pi} \frac{A_{i}}{k_{2}-a_{i}} \Pi_{a a}\left(k_{2}\right) \frac{A_{j}^{*}}{k_{2}-a_{j}^{*}} e^{-i k_{2} x} e^{i a_{j}^{*} y}
\end{aligned}
$$

and the fourth one is

$$
\begin{aligned}
& \sum_{i, j} \int \frac{d k_{2}}{2 \pi} \frac{A_{i}}{k_{2}-a_{i}} \Pi_{a a}\left(k_{2}\right) \frac{A_{j}^{*}}{k_{2}-a_{j}^{*}} e^{-i k_{2}(x-y)} \frac{1}{8}\left\{\theta\left(\epsilon_{1}-\epsilon_{2}\right)\left[1+\theta\left(\epsilon_{2}-\epsilon_{3}\right)\right]+\theta\left(\epsilon_{3}-\epsilon_{2}\right)\left[1+\theta\left(\epsilon_{2}-\epsilon_{1}\right)\right]\right\} \\
& \quad-\frac{1}{2} \sum_{i, j} \int \frac{d k_{2}}{2 \pi} \frac{A_{i}}{k_{2}-a_{i}} \Pi_{a a}\left(k_{2}\right) \frac{A_{j}^{*}}{k_{2}-a_{j}^{*}} e^{-i k_{2} x} e^{i a_{j}^{*} y}-\frac{1}{2} \sum_{i, j} \int \frac{d k_{2}}{2 \pi} \frac{A_{i}}{k_{2}-a_{i}} \Pi_{a a}\left(k_{2}\right) \frac{A_{j}^{*}}{k_{2}-a_{j}^{*}} e^{-i a_{i} x} e^{i k_{2} y} \\
& \quad+\sum_{i, j} \int \frac{d k_{2}}{2 \pi} \frac{A_{i}}{k_{2}-a_{i}} \Pi_{a a}\left(k_{2}\right) \frac{A_{j}^{*}}{k_{2}-a_{j}^{*}} e^{-i a_{i} x} e^{i a_{j}^{*} y} .
\end{aligned}
$$

Adding up the contributions in Eqs. (A14), (A16), (A17), and (A18) then gives that the contribution to $G_{r r}$ at the scale $\xi g \Lambda$ is

$$
\sum_{i, j} \int \frac{d k}{2 \pi} \frac{A_{i}}{k-a_{i}} \Pi_{a a}(k) \frac{A_{j}^{*}}{k-a_{j}^{*}}\left(e^{-i k x}-e^{-i a_{i} x}\right)\left(e^{i k y}-e^{i a_{j}^{*} y}\right) .
$$

The calculation for mixed terms with, say, contribution at scale $g \Lambda$ from the retarded correlator and contribution at scale $\xi g \Lambda$ from the advanced correlator proceeds analogously. The final result is precisely Eq. (39).

\section{APPENDIX B: EVALUATION OF FACTORS WITH MOMENTUM DISTRIBUTIONS}

We begin by showing Eq. (59), namely, that

$$
S_{1122}=2 F(P+K)(1-F(P)) \operatorname{Re} S_{\text {rraa }}
$$

in the presence of instabilities. Using that

$$
\phi_{1}=\phi_{r}+\frac{1}{2} \phi_{a}, \quad \phi_{2}=\phi_{r}-\frac{1}{2} \phi_{a}
$$

it is easy to see that

$$
\begin{aligned}
S_{1122}= & S_{\text {rrrr }}+\frac{1}{2}\left(S_{\text {arrr }}+S_{\text {rarr }}-S_{\text {rrar }}-S_{\text {rrra }}\right)+\frac{1}{4}\left(S_{\text {aarr }}-S_{\text {arar }}-S_{\text {arra }}-S_{\text {raar }}-S_{\text {rara }}+S_{\text {rraa }}\right) \\
& +\frac{1}{8}\left(-S_{\text {aaar }}-S_{\text {aara }}+S_{\text {araa }}+S_{\text {raaa }}\right)+\frac{1}{16} S_{\text {aaaa }} .
\end{aligned}
$$

The different four-point functions are defined in Fig. 9. Using that $a$ a propagators vanish we see that $S_{\text {naam }}=S_{\text {anma }}=0$ for any $n, m \in\{a, r\}$ so we are then left with

$$
S_{1122}=S_{\text {rrrr }}+\frac{1}{2}\left(S_{\text {arrr }}+S_{\text {rarr }}-S_{\text {rrar }}-S_{\text {rrra }}\right)+\frac{1}{4}\left(S_{\text {aarr }}-S_{\text {arar }}-S_{\text {rara }}+S_{\text {rraa }}\right) .
$$

Using Eq. (58) we furthermore see that $S_{r n m r}=\left[\frac{1}{2}-F(P)\right]\left(S_{\text {anmr }}-S_{\text {rnma }}\right)$ and $S_{n r r m}=\left[\frac{1}{2}-F(P+K)\right]\left(S_{\text {nram }}-S_{\text {narm }}\right)$ so

$$
\begin{aligned}
S_{1122}= & {\left[\frac{1}{2}-F(P+K)\right]\left[\frac{1}{2}-F(P)\right]\left(-S_{\text {rraa }}+S_{\text {arar }}+S_{\text {rara }}-S_{\text {aarr }}\right)+\left[\frac{1}{2}-F(P+K)\right] \frac{1}{2}\left(S_{\text {arar }}-S_{\text {aarr }}-S_{\text {rraa }}+S_{\text {rara }}\right) } \\
& +\left[\frac{1}{2}-F(P)\right] \frac{1}{2}\left(-S_{\text {rara }}+S_{\text {aarr }}+S_{\text {rraa }}-S_{\text {arar }}\right)+\frac{1}{4}\left(S_{\text {aarr }}-S_{\text {arar }}-S_{\text {rara }}+S_{\text {rraa }}\right) .
\end{aligned}
$$

We furthermore have that $S_{\text {arar }}=S_{\text {rara }}=0$ because the two quark propagators give theta functions of the form $\theta\left(t_{x}-t_{y}\right) \theta\left(t_{y}-\right.$ $\left.t_{x}\right)=0$. We are then left with

$$
S_{1122}=F(P+K)[1-F(P)]\left(S_{\text {rraa }}+S_{\text {aarr }}\right)=2 F(P+K)[1-F(P)] \operatorname{Re} S_{\text {rraa }} .
$$

We finally note how the momentum factors work out when there is a medium as well as background fields. Adding $n$ background field insertions to the bare $r r$ propagator gives

$$
S_{r r}^{(n)}\left(t_{x}, t_{y} ; \mathbf{p} ;\left\{\mathbf{l}_{1}, \ldots, \mathbf{l}_{n}\right\}\right)=\left[\frac{1}{2}-F(\mathbf{k})\right] \times\left[S_{\text {ret }}^{(n)}\left(t_{x}, t_{y} ; \mathbf{p} ;\left\{\mathbf{l}_{1}, \ldots, \mathbf{l}_{n}\right\}\right)-S_{\mathrm{adv}}^{(n)}\left(t_{x}, t_{y} ; \mathbf{p} ;\left\{\mathbf{l}_{1}, \ldots, \mathbf{l}_{n}\right\}\right)\right],
$$


as in Eq. (58). We can factor out the instability insertions to get

$$
S_{r r}^{(n)}\left(t_{x}, t_{y} ; \mathbf{p} ;\left\{\mathbf{l}_{1}, \ldots, \mathbf{l}_{n}\right\}\right)=\left[\frac{1}{2}-F(\mathbf{k})\right]\left[S_{\mathrm{ret}}^{(0)}\left(t_{x}, t_{y} ; \mathbf{p}\right)-S_{\mathrm{adv}}^{(0)}\left(t_{x}, t_{y} ; \mathbf{p}\right)\right]\left(i g A_{\mu} \widehat{K}^{\mu}\right)^{n} \prod_{j=1}^{n} \frac{e^{-i \widehat{\mathbf{k}} \cdot \mathbf{l}_{j}\left(t_{x}-t_{y}\right)}-1}{-i \widehat{\mathbf{k}} \cdot \mathbf{l}_{j}}
$$

or in other words

$$
S_{r r}^{(n)}\left(t_{x}, t_{y} ; \mathbf{p} ;\left\{\mathbf{l}_{1}, \ldots, \mathbf{l}_{n}\right\}\right)=S_{r r}^{(0)}\left(t_{x}, t_{y} ; \mathbf{p}\right)\left(i g A_{\mu} \widehat{K}^{\mu}\right)^{n} \prod_{j=1}^{n} \frac{e^{-i \widehat{\mathbf{k}} \cdot \mathbf{l}_{j}\left(t_{x}-t_{y}\right)}-1}{-i \widehat{\mathbf{k}} \cdot \mathbf{l}_{j}}
$$

for the $\widehat{P}$ part and similarly for the $\widetilde{P}$ part. The same argument as in Sec. VI then allows us to factor out the effect of background fields for any combination of $r r$, retarded, and advanced propagator and shows that the effect of the background field vanishes.

[1] B. V. Jacak and B. Muller, The exploration of hot nuclear matter, Science 337, 310 (2012).

[2] C. Gale, S. Jeon, and B. Schenke, Hydrodynamic modeling of heavy-ion collisions, Int. J. Mod. Phys. A 28, 1340011 (2013).

[3] R. Baier, Y. L. Dokshitzer, A. H. Mueller, S. Peigne, and D. Schiff, Radiative energy loss and $\mathrm{p}(\mathrm{T})$ broadening of highenergy partons in nuclei, Nucl. Phys. B 484, 265 (1997).

[4] A. Majumder, B. Muller, and X.-N. Wang, Small Shear Viscosity of a Quark-Gluon Plasma Implies Strong Jet Quenching, Phys. Rev. Lett. 99, 192301 (2007).

[5] G. D. Moore, Numerical studies of QGP instabilities and implications, Eur. Phys. J. A 29, 53 (2006).

[6] S. Mrówczyński, B. Schenke, and M. Strickland, Color instabilities in the quark-gluon plasma, Phys. Rep. 682, 1 (2017).

[7] M.E. Carrington, St. Mrówczyński, and B. Schenke, Momentum broadening in unstable quark-gluon plasma, Phys. Rev. C 95, 024906 (2017).

[8] M. E. Carrington, K. Deja, and S. Mrowczynski, Energy loss in unstable quark-gluon plasma, Phys. Rev. C 92, 044914 (2015).

[9] R. Baier and Y. Mehtar-Tani, Jet quenching and broadening: The transport coefficient q-hat in an anisotropic plasma, Phys. Rev. C 78, 064906 (2008).

[10] P. Romatschke, Momentum broadening in an anisotropic plasma, Phys. Rev. C 75, 014901 (2007).

[11] M. Nopoush, Y. Guo, and M. Strickland, The static hard-loop gluon propagator to all orders in anisotropy, J. High Energy Phys. 09 (2017) 063.

[12] P. B. Arnold, G. D. Moore, and L. G. Yaffe, Effective kinetic theory for high temperature gauge theories, J. High Energy Phys. 01 (2003) 030.

[13] S. Hauksson, S. Jeon, and C. Gale, Photon emission from quarkgluon plasma out of equilibrium, Phys. Rev. C 97, 014901 (2018).

[14] P. B. Arnold, G. D. Moore, and L. G. Yaffe, Photon emission from ultrarelativistic plasmas, J. High Energy Phys. 11 (2001) 057.

[15] J.-P. Blaizot and E. Iancu, The Quark gluon plasma: Collective dynamics and hard thermal loops, Phys. Rep. 359, 355 (2002).

[16] S. Mrówczyński and M. H. Thoma, Hard loop approach to anisotropic systems, Phys. Rev. D 62, 036011 (2000).

[17] P. Romatschke and M. Strickland, Collective modes of an anisotropic quark gluon plasma, Phys. Rev. D 68, 036004 (2003).

[18] P. B. Arnold, J. Lenaghan, and G. D. Moore, QCD plasma instabilities and bottom up thermalization, J. High Energy Phys. 08 (2003) 002.
[19] A. Rebhan and D. Steineder, Collective modes and instabilities in anisotropically expanding ultrarelativistic plasmas, Phys. Rev. D 81, 085044 (2010).

[20] R. Baier, M. Dirks, K. Redlich, and D. Schiff, Thermal photon production rate from nonequilibrium quantum field theory, Phys. Rev. D 56, 2548 (1997).

[21] B. Schenke and M. Strickland, Photon production from an anisotropic quark-gluon plasma, Phys. Rev. D 76, 025023 (2007).

[22] C. Shen, J.-F. Paquet, U. Heinz, and C. Gale, Photon emission from a momentum anisotropic quark-gluon plasma, Phys. Rev. C 91, 014908 (2015).

[23] P. B. Arnold, G. D. Moore, and L. G. Yaffe, Photon and gluon emission in relativistic plasmas, J. High Energy Phys. 06 (2002) 030.

[24] P. Aurenche, F. Gelis, and H. Zaraket, Landau-PomeranchukMigdal effect in thermal field theory, Phys. Rev. D 62, 096012 (2000).

[25] S. Jeon and G. D. Moore, Energy loss of leading partons in a thermal QCD medium, Phys. Rev. C 71, 034901 (2005).

[26] M. Strickland, J. Noronha, and G. S. Denicol, Anisotropic nonequilibrium hydrodynamic attractor, Phys. Rev. D 97, 036020 (2018).

[27] A. Kurkela and G. D. Moore, Thermalization in weakly coupled nonabelian plasmas, J. High Energy Phys. 12 (2011) 044.

[28] J. Berges, K. Boguslavski, S. Schlichting, and R. Venugopalan, Turbulent thermalization process in heavy-ion collisions at ultrarelativistic energies, Phys. Rev. D 89, 074011 (2014).

[29] J. Berges, K. Boguslavski, S. Schlichting, and R. Venugopalan, Universal attractor in a highly occupied non-Abelian plasma, Phys. Rev. D 89, 114007 (2014).

[30] S. Hauksson, S. Jeon, and C. Gale, Penetrating probes: Jets and photons in a non-equilibrium quark-gluon plasma, Nucl. Phys. A 982, 787 (2019).

[31] S. Hauksson, S. Jeon, and C. Gale (unpublished).

[32] M. L. Bellac, Thermal Field Theory, Cambridge Monographs on Mathematical Physics (Cambridge University Press, Cambridge, England, 2011).

[33] J. Berges, Introduction to nonequilibrium quantum field theory, AIP Conf. Proc. 739, 3 (2004).

[34] P. Romatschke and M. Strickland, Collective modes of an anisotropic quark-gluon plasma II, Phys. Rev. D 70, 116006 (2004).

[35] I. Dadić, D. Klabučar, and D. Kuić, Direct photons from hot quark matter in renormalized finite-time-path QED, Particles 3, 676 (2020). 